ARTICLE

Received 8 Sep 2014 | Accepted 5 Feb 2015 | Published 13 Mar 2015

DOI: $10.1038 /$ ncomms7537

OPEN

\title{
The interferon-related developmental regulator 1 is used by human papillomavirus to suppress NFKB activation
}

Bart Tummers ${ }^{1}$, Renske Goedemans ${ }^{1}$, Laetitia P.L. Pelascini ${ }^{2}$, Ekaterina S. Jordanova ${ }^{3}$, Edith M.G. van Esch ${ }^{4}$, Craig Meyers ${ }^{5}$, Cornelis J.M. Melief ${ }^{6}$, Judith M. Boer ${ }^{7, \dagger} \&$ Sjoerd H. van der Burg ${ }^{1}$

High-risk human papillomaviruses (hrHPVs) infect keratinocytes and successfully evade host immunity despite the fact that keratinocytes are well equipped to respond to innate and adaptive immune signals. Using non-infected and freshly established or persistent hrHPVinfected keratinocytes we show that hrHPV impairs the acetylation of NFKB/RelA K310 in keratinocytes. As a consequence, keratinocytes display a decreased pro-inflammatory cytokine production and immune cell attraction in response to stimuli of the innate or adaptive immune pathways. HPV accomplishes this by augmenting the expression of interferon-related developmental regulator 1 (IFRD1) in an EGFR-dependent manner. Restoration of NFKB/RelA acetylation by IFRD1 shRNA, cetuximab treatment or the HDAC1/3 inhibitor entinostat increases basal and induced cytokine expression. Similar observations are made in IFRD1-overexpressing HPV-induced cancer cells. Thus, our study reveals an EGFR-IFRD1-mediated viral immune evasion mechanism, which can also be exploited by cancer cells.

\footnotetext{
${ }^{1}$ Department of Clinical Oncology, Leiden University Medical Center, Albinusdreef 2, 2333ZA Leiden, The Netherlands. ${ }^{2}$ Department of Molecular Cell Biology, Leiden University Medical Center, Albinusdreef 2, 2333ZA Leiden, The Netherlands. ${ }^{3}$ Center for Gynaecological Oncology, Plesmanlaan 121, 1066CX Amsterdam, The Netherlands. ${ }^{4}$ Department of Gynaecology, Leiden University Medical Center, Albinusdreef 2, 2333ZA Leiden, The Netherlands.

${ }^{5}$ Department of Microbiology and Immunology, The Pennsylvania State University College of Medicine, 500 University Drive, Hershey, Pennsylvania 17033, USA. ${ }^{6}$ Department of Immunohematology and Blood Transfusion, Leiden University Medical Center, Albinusdreef 2, 2333ZA Leiden, The Netherlands.

7 Department of Human Genetics, Leiden University Medical Center, Albinusdreef 2, 2333ZA Leiden, The Netherlands. $†$ Present addresses: Department of Pediatric Oncology, Erasmus MC-Sophia Children's Hospital, Wytemaweg 80, 3015CN Rotterdam, The Netherlands; Netherlands Bioinformatics Center, Geert Grooteplein-Zuid 28, 6525GA Nijmegen, The Netherlands. Correspondence and requests for materials should be addressed to S.H.v.d.B. (email: shvdburg@lumc.nl).
} 
igh-risk human papillomaviruses (hrHPVs) are absolutely species-specific small double-stranded DNA viruses that primarily target undifferentiated keratinocytes (KCs) of squamous epithelia via micro-wounds and abrasions. HrHPV infections can last up to 2 years despite viral activity in infected $\mathrm{KCs}$, the expression of viral antigens and the presence of KC-expressed pattern recognition receptors $(\mathrm{PRRs})^{1-4}$ that should lead to activation of innate and adaptive immune responses. This indicates that hrHPV has evolved mechanisms to transiently evade innate and adaptive immune mechanisms. Ultimately, the majority of hrHPV infections are controlled by the immune system, in particular by type-1 interferon (IFN)- $\gamma$ and tumour necrosis factor (TNF)- $\alpha$ cytokine-producing $\mathrm{T}$ cells ${ }^{5}$. In case of immune failure, hrHPV causes cancer of the anogenital and/or head and neck regions ${ }^{6}$.

Upon infection, hrHPV alters the immune-related response of KCs to various innate and adaptive immune stimuli, resulting in impaired expression of IFN-stimulated genes, interferon regulatory transcription factor-induced genes and $\mathrm{NF \kappa B}$-induced genes $^{3,7-12}$, suggesting that HPV hampers STAT1 and NFKB activation. HPV-infected KCs display downregulated basal expression of STAT1 and lowered STAT1 protein levels, explaining the impaired expression of IFN-stimulated genes ${ }^{13-16}$. Furthermore, soon after infection HPV upregulates the cellular deubiquitinase ubiquitin carboxy-terminal hydrolase L1 (UCHL1) to impair PRR-induced NFKB activation by upstream interference with TRAF3, TRAF6 and $\mathrm{NEMO}^{8}$. The upregulation of UCHL1, however, cannot explain how the virus manages to suppress the KCs response to adaptive immune signals ${ }^{12}$. In addition, repressing UCHL1 does not fully restore NFKB signalling via $\mathrm{PRR}^{8}$, suggesting that one or more additional mechanisms are in play to suppress NFKB signalling.

In this study, we analyse NFKB activation and subsequent cytokine/chemokine production following IFN- $\gamma$ and TNF- $\alpha$ stimulation in uninfected and HPV-infected primary KCs. Our study reveals that RelA acetylation, needed for NFKB transcriptional activity ${ }^{17}$, is impaired in hrHPV-infected KCs. The HPVinduced overexpression of the cellular protein interferon-related developmental regulator 1 (IFRD1) is shown to be instrumental in this process and involves histone deacetylases (HDACs) 1 and/or 3. The augmented expression of IFRD1 is the result of the HPV-mediated upregulation of the epidermal growth factor receptor (EGFR). Blocking of IFRD1 protein expression by small hairpin RNA (shRNA) or via the anti-EGFR antibody cetuximab restores $\mathrm{NF \kappa B} /$ RelA-mediated cytokine expression. Additional data suggest that IFRD1 may have a similar role in suppressing cytokine/chemokine production in HPV-positive cervical cancer cells.

\section{Results}

HrHPV impairs the KCs cytokine response to IFN- $\gamma$ and TNF- $\alpha$. To evaluate whether the KCs immune response following the exposure to IFN- $\gamma$ and/or TNF- $\alpha$ is attenuated by hrHPV, we utilized a system that resembles the natural infection with hrHPV as closely as possible. Primary KCs stably maintaining the hrHPV genome as episomes (hrHPV $+\mathrm{KCs}$ ) display similar growth properties as non-transfected KCs, and upon culture in organotypic raft cultures, mimic HPV infection in vivo as documented by genome amplification, late gene expression and virus production during the differentiation-dependent life cycle of $\mathrm{HPV}^{18-20}$.

The presence of HPV type 16 (HPV16) was clearly associated with an impaired capacity to respond to IFN- $\gamma$ and to TNF- $\alpha$, as shown by the lower messenger RNA (mRNA) expression and production of the IFN- $\gamma$ and/or TNF- $\alpha$-induced pro-inflammatory cytokines CCL2, RANTES (CCL5), interleukin (IL)-8 and the chemokines CXCL9, 10 and 11 by KCs (Fig. 1a,b). Not only did the presence of HPV16 impair the production of cytokines, also the migration of peripheral blood mononuclear cells (PBMCs) to supernatants of IFN- $\gamma$ and TNF- $\alpha$-stimulated HPV16 + KCs was greatly impaired (Fig. 1c).

These data suggest that hrHPV, besides impairing the innate immune response of $\mathrm{KCs}^{8}$, also suppresses the $\mathrm{KCs}$ response to the adaptive immune signals provided by IFN- $\gamma$ and TNF- $\alpha$.

The hrHPV-mediated deregulated expression of STAT1 (refs 13-16) may explain the impaired cytokine expression by hrHPV-positive KCs upon IFN- $\gamma$ stimulation, but not the impaired response to TNF- $\alpha$ (IL-8) or to IFN- $\gamma$ and TNF- $\alpha$ (RANTES). Previously, we showed that hrHPV hampers phosphorylation of the NFKB subunit RelA (p65) upon stimulation with the innate PRR ligand poly $(\mathrm{I}: \mathrm{C})^{8}$. As TNF- $\alpha$ stimulation rapidly induces the phosphorylation of $\operatorname{RelA}^{17}$, we tested whether hrHPV also hampers rapid TNF- $\alpha$-induced RelA phosphorylation by stimulating KCs and HPV16 + KCs for 0, 5, 15 or 30 min with TNF- $\alpha$. Western blotting showed that RelA was rapidly phosphorylated similarly in KCs and HPV16+KCs, peaking after $15 \mathrm{~min}$ of TNF- $\alpha$ stimulation (Fig. 1d), indicating that the impairment of TNF- $\alpha$-induced responses seen in HPV16 + KCs was not due to altered RelA phosphorylation after short-term TNF- $\alpha$ stimulation. Activated NFKB translocates to the nucleus where it is modified to regulate its DNA-binding ability and transcriptional activity. Acetylation of the RelA subunit at lysine $310(\mathrm{~K} 310)$ is crucial in this process ${ }^{17}$. Strikingly, acetylated RelA K310 protein levels were lower in the HPV16 + KCs than in uninfected KCs, both in the absence of stimulation and after short-term TNF- $\alpha$ stimulation (Fig. 1d). The lowered basal RelA K310 acetylation state was verified in three independent primary $\mathrm{KC}$ and two independent HPV16 + KC cultures (Fig. 1e), indicating that HPV hampers the activity of NFKB already at steady-state levels. This was also reflected in a lowered basal cytokine gene expression in unstimulated HPV16 + KCs (Fig. 1f).

HrHPV upregulates IFRD1 to impair RelA K310 acetylation. Acetylation of RelA K310 can be regulated by the lysine acetyl transferases (KAT) PCAF (KAT2B), CREBBP (KAT3A), p300 (KAT3B) and TIP60 (KAT5), as well as the HDACs 1 and 3 (ref. 17). Since our results imply that hrHPV has a mechanism either to deacetylate or impair the acetylation of RelA, we screened our validated microarray data ${ }^{12}$ for genes involved in regulating RelA K310 acetylation. HrHPV did not significantly influence HDAC (HDAC1 to 11) or sirtuin (SIRT1 to 7) expression (Fig. 2a). The only significantly upregulated gene was the lysine acetyl transferase CREBBP (KAT3A), confirming previous observations stating that HPV upregulates CREBBP to enhance expression from episomal DNA ${ }^{21,22}$. However, as CREBBP acetylates RelA its upregulation cannot explain the observed lower levels of RelA K310 acetylation in hrHPV-infected $\mathrm{KCs}$ under steady-state conditions. Interestingly, the microarray data also showed the upregulation of IFRD1 (Fig. 2b), which previously was shown to complex HDAC1 (ref. 23) and HDAC3 to RelA causing its deacetylation at lysine 310 in the mouse myoblast cell line $\mathrm{C} 2 \mathrm{C} 12$ (ref. 24). We hypothesized that it may fulfil a similar role in human KCs. Therefore, reverse transcriptase (RT)-quantitative PCR (qPCR) and western blotting was used to confirm that IFRD1 gene expression (Fig. 2c, left) and IFRD1 protein levels (Fig. 2d, left) were elevated in two independent HPV16 + KC cultures. Knockdown of the polycistronic mRNA of HPV16 by a small interfering RNA (siRNA) against HPV16 E2 in HPV16+KCs resulted in the reduction of HPV16 E1, E2, E6 and E7 expression 
(Supplementary Fig. 1), IFRD1 mRNA (Fig. 2c, middle) and IFRD1 protein levels (Fig. 2d, middle), indicating that the augmented IFRD1 levels in hrHPV $+\mathrm{KCs}$ are the result of the presence of hrHPV. Reciprocally, when undifferentiated KCs were infected with native HPV16 virions, IFRD1 mRNA (Fig. 2c, right) and IFRD1 protein (Fig. 2d, right) levels were clearly enhanced after 2 days of infection. Furthermore, immunohistochemistry of HPV-positive vulvar lesions revealed the presence of IFRD1 in the nuclei of cells positive for HPV16 E2 (reflecting HPV-infected cells) ${ }^{25}$, but not in the nuclei of already transformed KCs (identified through p16 staining 25,26 or undifferentiated (E2 and p16 negative) healthy tissue (Fig. 2e).
We then asked whether the hrHPV-induced increased levels of IFRD1 affected RelA K310 acetylation also in human undifferentiated KCs. Indeed, when lentivirus-delivered siRNA against IFRD1 was used to lower IFRD1 protein expression, a concomitant increase in the steady-state levels of acetylated RelA K310 in HPV16 + KCs was seen when compared with control knockdown HPV16 + KCs (Fig. 2f; Supplementary Fig. 2a). Furthermore, a small increase in total RelA protein levels was observed. The gain in acetylated RelA K310 translated into a higher basal expression and secretion of cytokines in IFRD1 KD cells (Fig. 2g-i), indicating that IFRD1 is involved in the deregulation of steady-state inflammatory gene expression levels
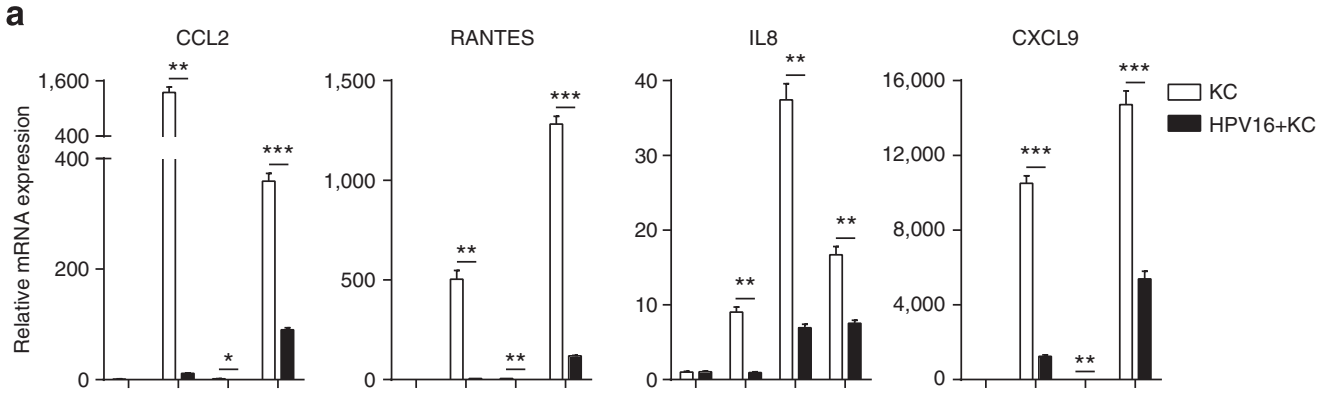

b
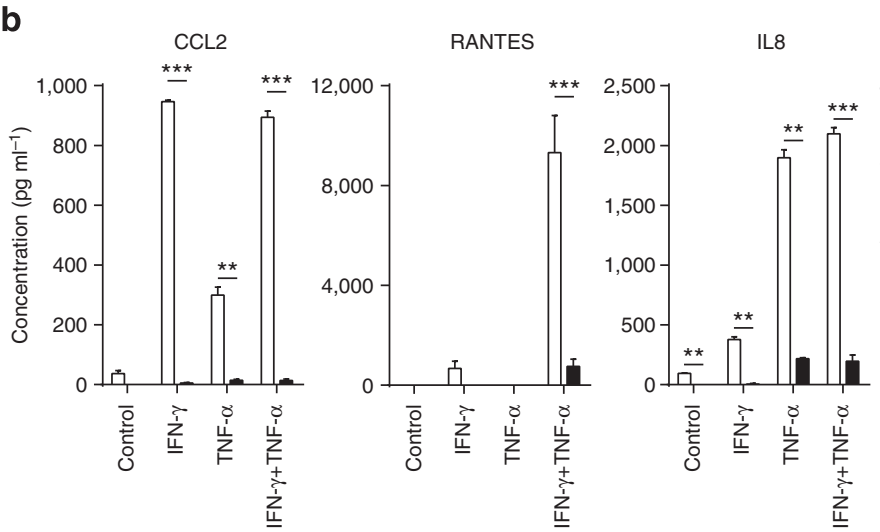

e

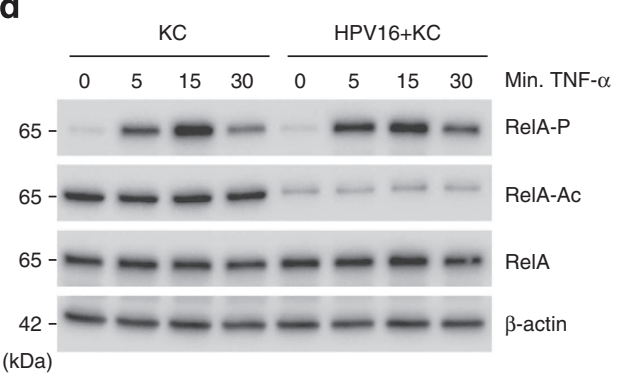

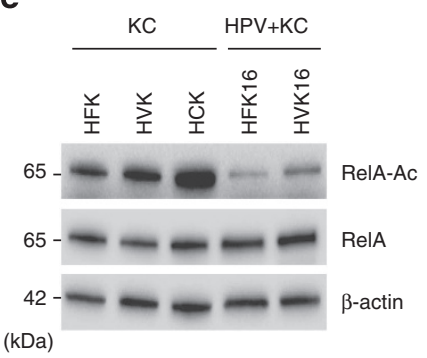

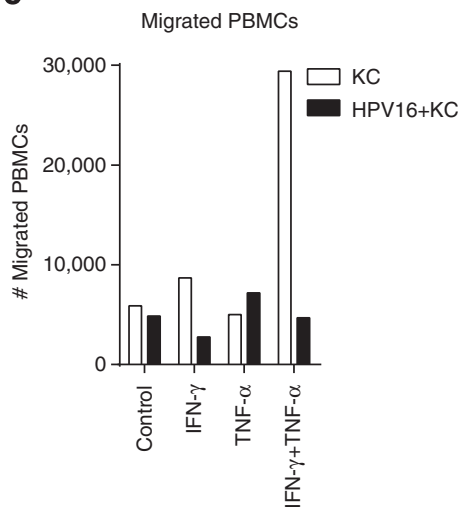

f

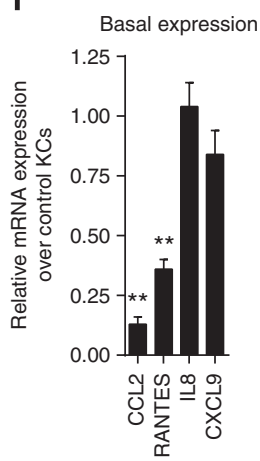

Figure 1 | HPV16 impairs IFN- $\gamma$ and TNF- $\alpha$-induced cytokine production and RelA K310 acetylation in KCs. (a) RT-qPCR of CCL2, RANTES, LL-8 and CXCL9 expression by 24-h control, IFN- $\gamma$ - and/or TNF- $\alpha$-stimulated undifferentiated KCs or HPV16 + KCs. Gene expression was normalized using GAPDH as the calibrator gene. Fold changes over control-stimulated undifferentiated KCs were calculated and depicted. (b) Enzyme-linked immunosorbent assay for CCL2, RANTES, IL-8 and CXCL9 in cleared supernatants of 24-h control, IFN- $\gamma$ - and/or TNF- $\alpha$-stimulated undifferentiated KCs or HPV16+KCs. (c) PBMCs migration towards cleared supernatants of 24-h control, IFN- $\gamma$ - and/or TNF- $\alpha$-stimulated KCs or HPV16 + KCs. A representative example of three different donors is shown. (d) RelA phosphorylation, acetylation and total levels in KCs and HPV16+KCs stimulated with TNF- $\alpha$ for 0,5 , 15 and $30 \mathrm{~min}$. (e) RelA acetylation and total levels at steady state in three human primary KC donor pools originating from human foreskin keratinocytes (HFKs), human vaginal keratinocytes (HVKs) or human cervical keratinocytes (HCKs) and two HPV16 + genome-transfected primary KC pools of foreskin (HFK16) or vaginal (HVK16) origin. (f) RT-qPCR of CCL2, RANTES, IL-8 and CXCL9 in HPV16 + KCs and KCs. Gene expression was normalized using GAPDH as the calibrator gene. Gene expression in HPV16 + KCs was standardized over KCs. All data are representative for at least three independent experiments. Error bars indicate s.d. $P$ values were determined using Welch-corrected unpaired $t$-tests. ${ }^{\star} P<0.05,{ }^{\star \star} P<0.01$ and ${ }^{\star \star \star} P<0.001$. 
in HPV16 + KCs. The dampening effect of IFRD1 on the NFKBregulated cytokine expression became even more apparent when the KCs were stimulated with both IFN- $\gamma$ and TNF- $\alpha$ (Fig. 2h,i). The cytokine levels produced after stimulation were much higher in IFRD1 KD HPV16 + KCs than in control KD HPV16+ KCs.
Moreover, IFRD1 knockdown augmented the ability of HPV16+ $\mathrm{KCs}$ to attract PBMCs (Fig. 2j). The main results were recapitulated in HPV18-infected KCs (Supplementary Fig. 3), suggesting that IFRD1 may form a general mechanism exploited by any hrHPV type.

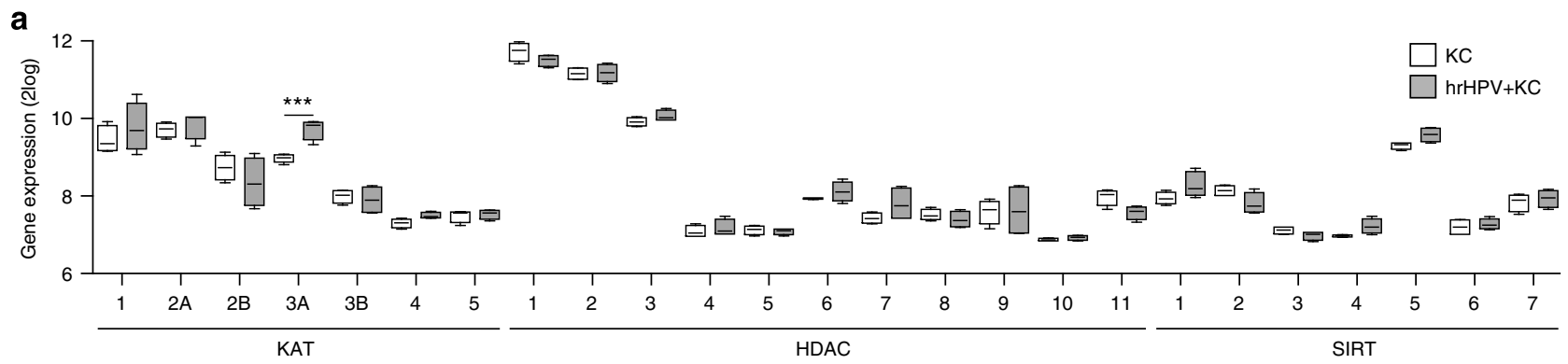

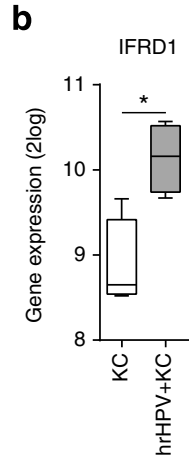

e

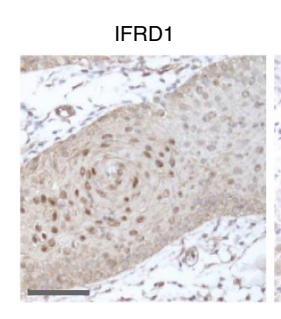

\section{C}

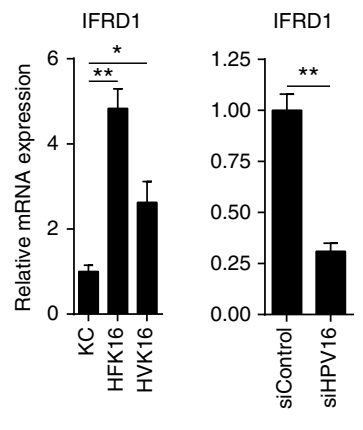

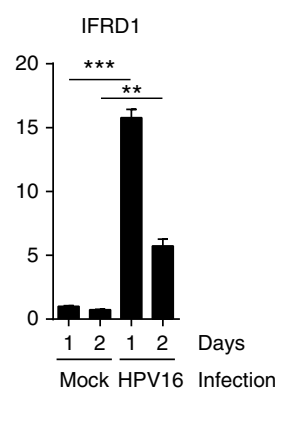

d

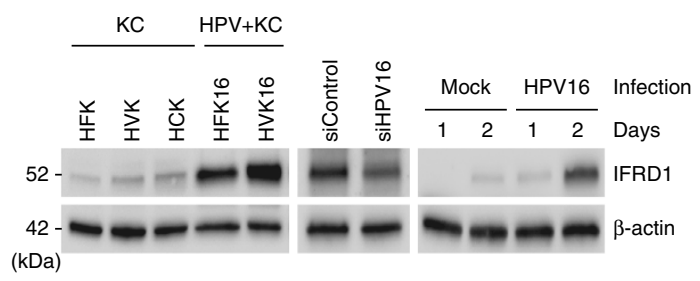

f

E2
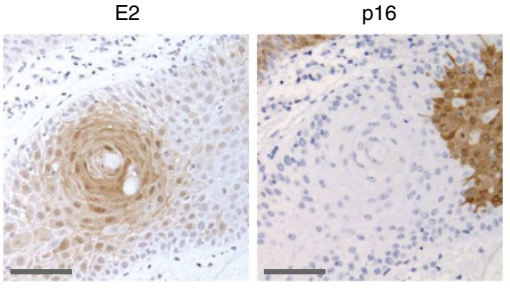

Control

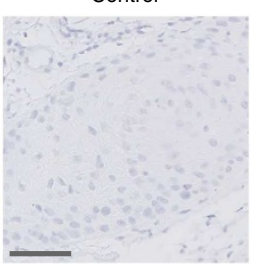

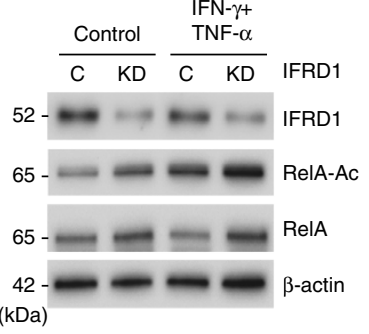

g

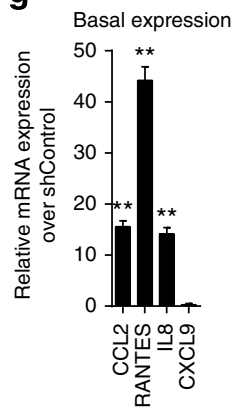

h
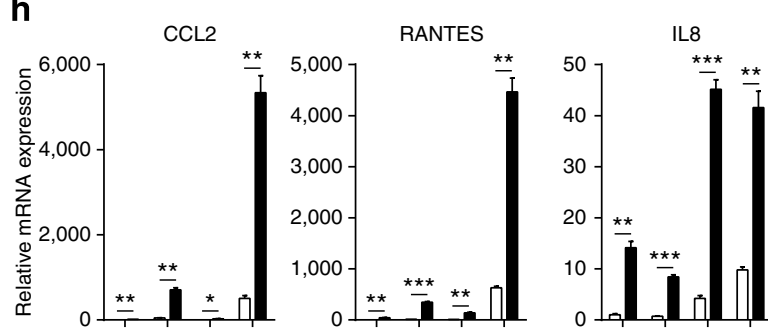

CXCL9

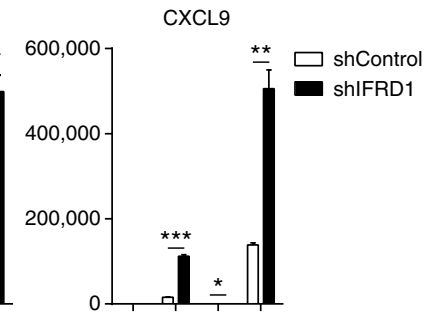

i
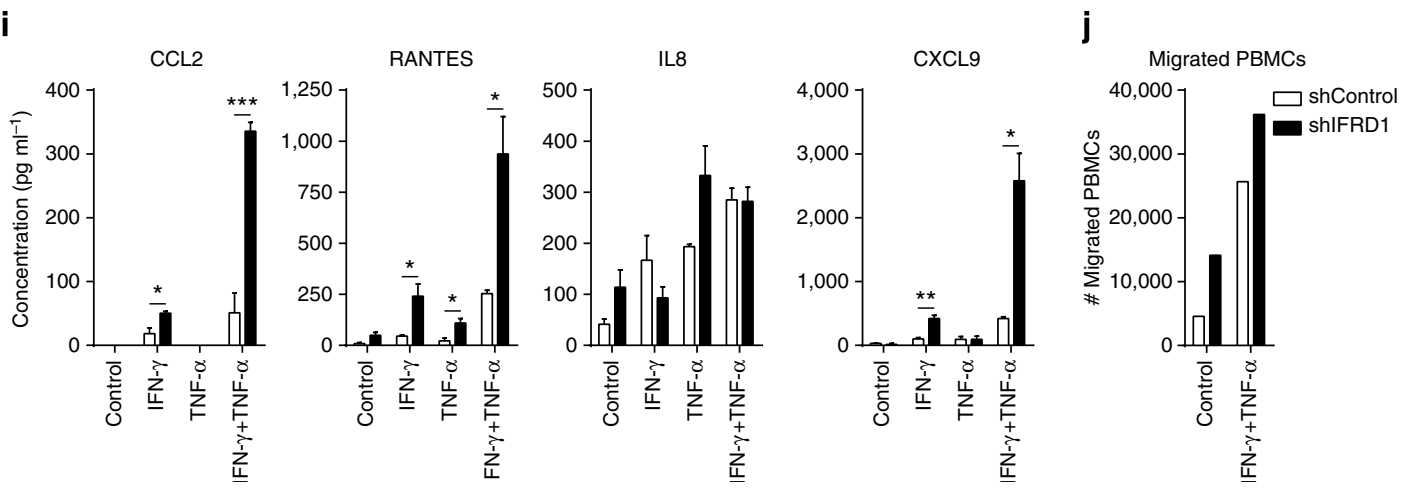
As the effect of IFRD1 occurred directly at the level of RelA, the influence of IFRD1 on the response of HPV16+KCs to poly(I:C) stimulation, previously shown by us to be impaired in hrHPV-infected $\mathrm{KCs}^{8}$, was also tested. Knockdown of IFRD1 resulted in an enhanced expression of CCL2, RANTES, IL-8 and CXCL9 following PRR stimulation with poly(I:C) (Supplementary Fig. 4).

Thus, hrHPV upregulates the expression of IFRD1 soon after infection, thereby effectively decreasing the basal levels of transcriptionally active RelA and as a consequence the levels of pro-inflammatory cytokines induced via various innate and adaptive immune-mediated NFKB stimulatory pathways.

EGFR signalling mediates the increased expression of IFRD1. Growth factors, such as nerve growth factor, fibroblast growth factor or EGF, have previously been shown to induce the expression of Tis family genes, which also includes IFRD1, in rat neocortical astrocytes and chromaffin cell line PC12, mouse C243 and IEC-18, and mammary epithelial cells ${ }^{27}$. The hrHPV E5 protein is known to affect different aspects of EGFR signalling and expression ${ }^{28}$. Verification of EGFR expression in our model showed that EGFR mRNA expression (Fig. 3a) and membranebound protein expression (Fig. 3b) were higher in hrHPV + KCs than in non-infected KCs. When we transfected complementary DNA for E2 (as control), E5 or a mix of several other HPV proteins, only E5 enhanced EGFR expression (Fig. 3c). To test whether EGFR signalling had a similar effect on IFRD1 in human primary KCs, the clinically used anti-EGFR antibody cetuximab was employed to block EGFR signalling. Indeed, IFRD1 expression decreased in HPV16 + KCs, but not in uninfected KCs, when treated with cetuximab (Fig. 3d). IFRD1 protein levels also decreased dose-dependently in both cetuximab-treated noninfected KCs and HPV16 + KCs (Fig. 3e). Notably, the isotype control antibody rituximab (anti-CD20) had no effect (Fig. 3d,e). Thus EGFR signalling does not only induce IFRD1 gene expression but also stabilizes IFRD1 protein levels. Relative density analysis revealed that in cetuximab-treated HPV16 + KCs the protein levels of IFRD1 decreased while concomitantly the levels of RelA K310 acetylation increased in a dose-dependent fashion. Total RelA levels were unaffected (Fig. 3f). These results indicated that the HPV-induced expression of IFRD1 is mediated via the EGFR signalling pathway, and implied that cetuximab treatment may enhance the hrHPV + KCs pro-inflammatory cytokine response to immune stimuli. Indeed, upon IFN- $\gamma$ and TNF- $\alpha$ stimulation cetuximab-treated HPV16 + KCs expressed higher levels of indicated cytokine genes than rituximab-treated cells (Fig. 3g), as well as higher levels of secreted cytokines
(Fig. 3h). In uninfected KCs, treatment with cetuximab decreased the already low levels of IFRD1 protein, and although this led to increased cytokine gene expression after IFN- $\gamma$ and TNF- $\alpha$ stimulation, no additional increase in the already high levels of secreted cytokines was observed (Fig. 3g,h). The absence of cytokine production in cetuximab-treated $\mathrm{HPV} 16+\mathrm{KC}$ and uninfected KCs that were not stimulated with IFN- $\gamma$ and TNF- $\alpha$ shows that binding of cetuximab to EGFR per se does not result in the stimulation of cytokine production (Fig. 3g,h).

As EGFR signalling involves the downstream partners PI3K, mTOR, MEK1, RAF and JNK, we selectively inhibited these proteins using small-molecule inhibitors in HPV16+KCs and observed that selective inhibition of mTOR (rapamycin), MEK1 (PD98059) and RAF (GW5074), but not PI3K (LY94002) or JNK (SP60025), resulted in decreased expression of IFRD1 (Fig. 3i). Thus EGFR-mediated upregulation of IFRD1 is fundamental to the impaired NFKB-induced cytokine response of hrHPV-infected KCs to innate and adaptive immune stimuli.

HDAC1/3 inhibition stimulates cytokine production. IFRD1mediated RelA deacetylation required the recruitment of HDAC1 and/or -3 to the RelA-IFRD1 complex in the mouse myoblast cell line $\mathrm{C} 2 \mathrm{C} 12$ (ref. 24). To test whether these HDACs played a similar role in human hrHPV + KCs, the effect of HDAC inhibition was tested in HPV16 + KCs and non-infected KCs. A dose titration of the HDAC1/3-specific inhibitor entinostat (MS-275), and the prototypic pan-HDAC inhibitors trichostatin A, sodium butyrate $(\mathrm{NaBu})$ and the Food and Drug Administrationapproved vorinostat (suberoylanilide hydroxamic acid (SAHA)) was performed to study RelA K310 acetylation. All pan-HDAC inhibitors increased RelA acetylation in KCs at the lowest concentration used (Fig. 4a; Supplementary Fig. 2b), but at higher doses cells suffered from toxic effects as observed by microscopy. However, HPV16 + KCs did survive entinostat treatment, and clearly this HDAC1/3 inhibitor increased RelA K310 acetylation in HPV16 + KCs (Fig. 4a; Supplementary Fig. 2b). This indicated that HDAC1 and/or -3 are indeed specifically involved in the deacetylation of RelA in hrHPV + KCs. Entinostat treatment of HPV16 + KCs not only restored RelA K310 acetylation but also released the suppressive effect of IFRD1 on cytokine production. Treated HPV16 + KCs displayed a higher basal expression for three out of four tested cytokines when compared with their untreated counterparts (Fig. 4b). Moreover, when stimulated with IFN- $\gamma$ and TNF- $\alpha$ both KCs and HPV16 + KCs displayed a higher expression of CCL2, IL-8 and CXCL9, although the expression of RANTES was abrogated (Fig. 4c). To confirm the

Figure 2 | HrHPV upregulates IFRD1 to impair RelA K310 acetylation and basal cytokine expression. Microarray intensities for (a) all KATS, HDACs and SIRTs, and (b) IFRD1 in four independent KCs and four independent hrHPV + KCs represented in a box plot. The box contains the 1st quartile up to the 3rd quartile; the median is represented as a line; whiskers represent the values of the outer two quartiles. (c) IFRD1 mRNA expression of one representative control primary KC culture and two HPV16 + KC cultures (left panel), in HFK16 cells transfected with siControl or siHPV16 (middle panel) and in primary $\mathrm{KCs}$ that are either mock infected or infected with native HPV16 virions (right panel), as measured by RT-qPCR. (d) IFRD1 protein expression in three human primary keratinocyte (KC) donor pools originating from human foreskin keratinocytes (HFKs), human vaginal keratinocytes (HVKs) or human cervical keratinocytes (HCKs) and two HPV16 + genome-transfected primary KC pools of foreskin (HFK16) or vaginal (HVK16) origin (left panel) in HFK16 cells transfected with siControl or siHPV16 (middle panel) and in primary KCs that are either mock infected or infected with native HPV16 virions (right panel), as measured by western blot. (e) Immunohistochemical staining for IFRD1, HPV16 E2, p16 and negative antibody control of a vulvar intraepithelial neoplasia (VIN) lesion, one representative donor of two shown. Counterstaining was done using haematoxylin. Scale bar, 500 $\mu \mathrm{m}$. (f) IFRD1, RelA K310 acetylation and total RelA levels in 24-h non- or IFN- $\gamma$ - and TNF- $\alpha$-stimulated control or IFRD1 knockdown (KD) HPV16 + KCs. (g) RT-qPCR of CCL2, RANTES, IL-8 and CXCL9 expression in steady-state control or IFRD1 KD HPV16 + KCs. (h) RT-qPCR of CCL2, RANTES, IL-8 and CXCL9 expression in 24-h non- or IFN- $\gamma$ - and/or TNF- $\alpha$-stimulated control or IFRD1 KD HPV16 + KCs. (i) Enzyme-linked immunosorbent assay for CCL2, RANTES, IL-8 and CXCL9 in cleared supernatants of 24-h non- or IFN- $\gamma$ - and/or TNF- $\alpha$-stimulated control or IFRD1 KD HPV16 + KCs. (j) PBMCs migration towards cleared supernatants of 24-h non- or IFN- $\gamma$ - and TNF- $\alpha$-stimulated control or IFRD1 KD HPV16 + KCs. A representative example of three different donors is shown. These data are representative for at least three independent experiments. Error bars indicate s.d. $P$ values were determined using Welch-corrected unpaired t-tests. ${ }^{\star} P<0.05,{ }^{\star \star} P<0.01$ and ${ }^{\star \star \star} P<0.001$. 
involvement of RelA in this process, RelA was knocked down in $\mathrm{HPV}+\mathrm{KCs}$ (Fig. 4d), after which the cells were treated with entinostat and stimulated with IFN- $\gamma$ and TNF- $\alpha$. Indeed, RelA acetylation and cytokine production was increased in the control knockdown cells after stimulation with IFN- $\gamma$ and TNF- $\alpha$ when treated with entinostat (Fig. 4d,e). However, when RelA was knocked down in HPV16 + KCs, the cytokine expression was abrogated despite treatment with entinostat (Fig. 4e).

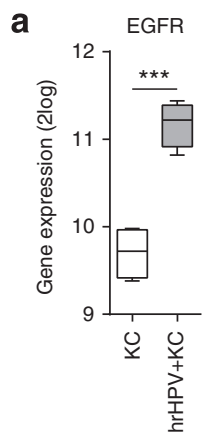

b

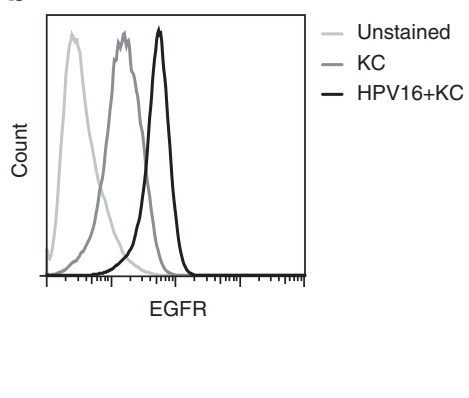

e

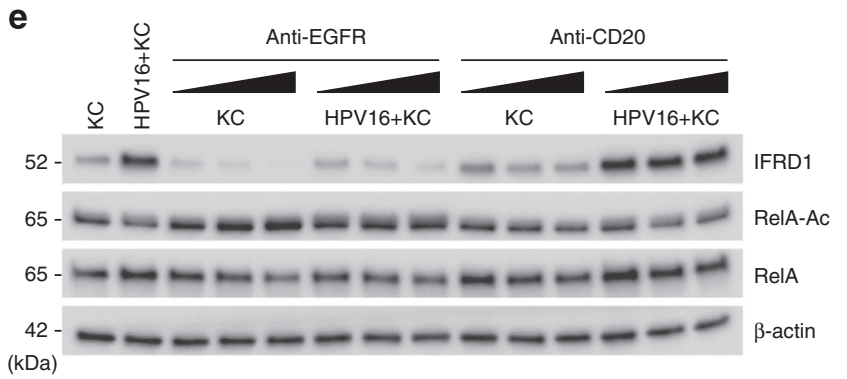

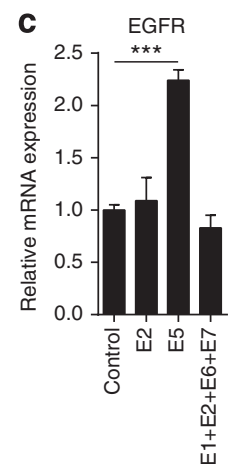

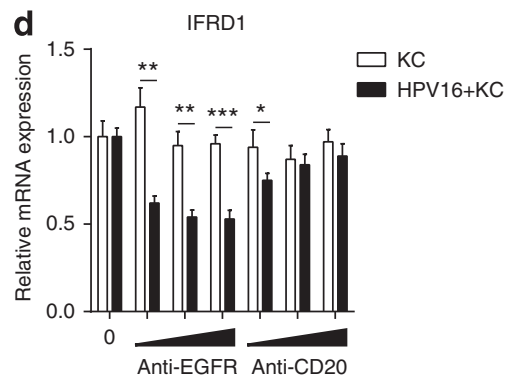

g

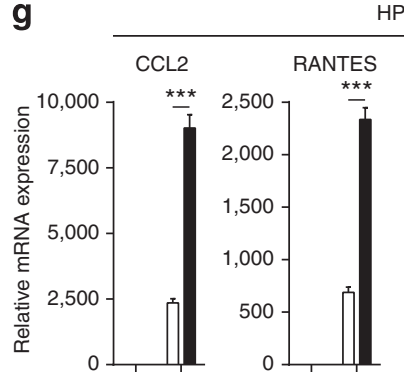

HPV16+KC
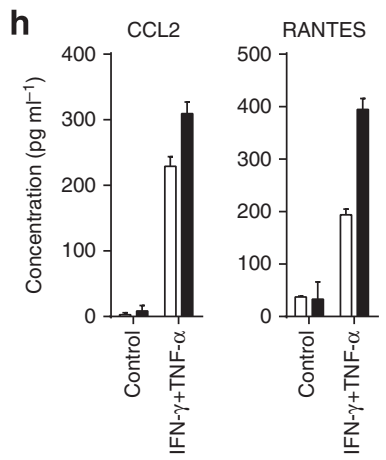
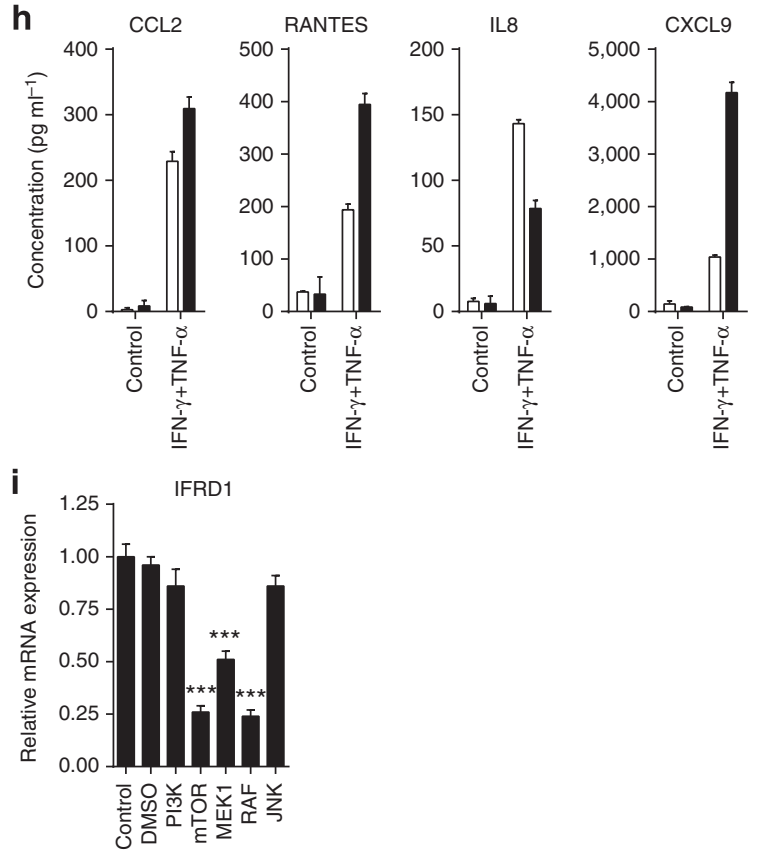

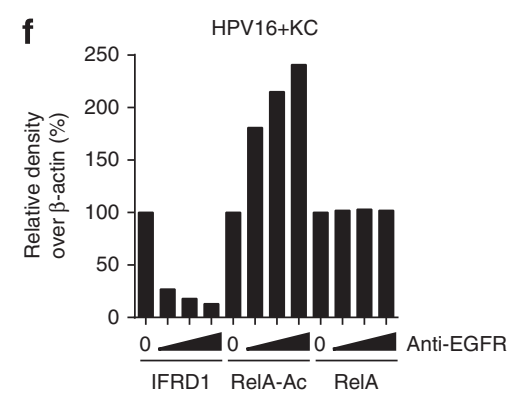

$\mathrm{KC}$
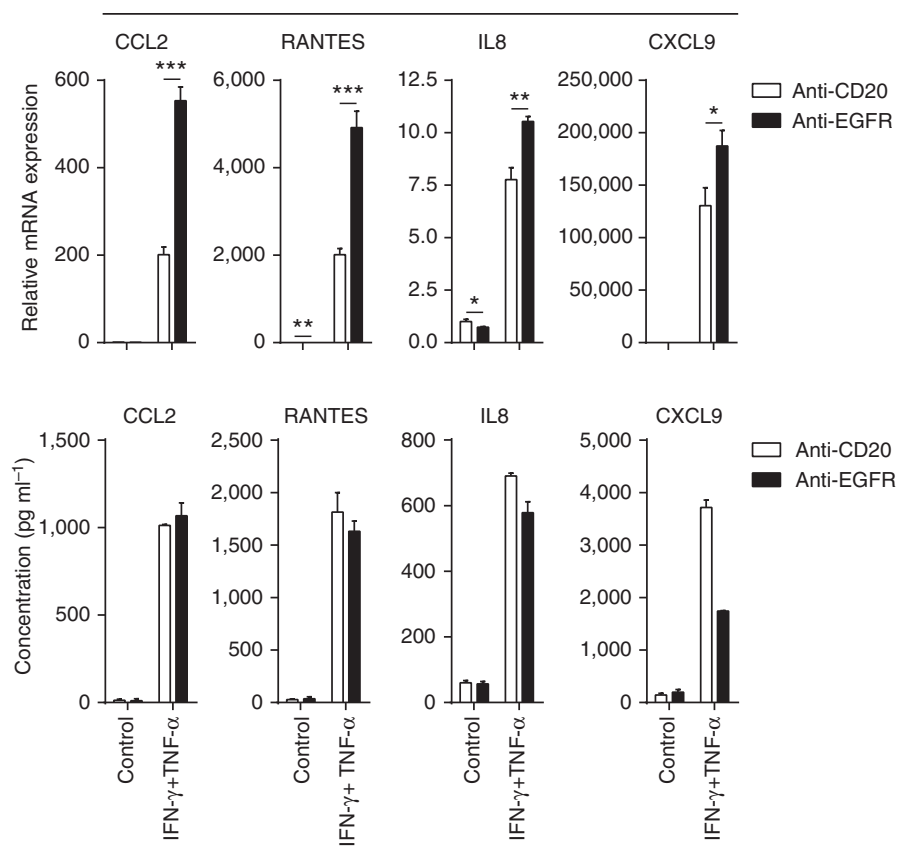
Previously, it was shown that HDAC inhibition abrogates EGFR expression ${ }^{29,30}$, indicating that EGFR expression is dependent on acetylation events. Indeed, entinostat treatment dose-dependently abrogated EGFR expression in hrHPV $+\mathrm{KCs}$, but did not influence the expression in KCs (Fig. 4f). Furthermore, entinostat treatment resulted in a reduced level of IFRD1 protein in hrHPV + KCs (Fig. 4g), which made us wonder whether IFRD1 could regulate EGFR expression. Therefore, IFRD1 was knocked down in hrHPV + KCs and this resulted in lower EGFR expression (Fig. 4h) and a lower level of membranebound EGFR than control-treated hrHPV + KCs (Fig. 4i,j), indicating that IFRD1 can control EGFR expression.

IFRD1 hampers the response of cancer cells to IFN- $\gamma$ and TNF- $\alpha$. To evaluate whether an increased expression of IFRD1 could also play a role in HPV16-induced squamous cell carcinoma, we analysed the cell line Caski, as well as the two early-passage cervical cancer cell lines, CSCC1 and CSCC7 (ref. 31). IFRD1 protein expression differed between the cell lines (Fig. 5a), but was increased in Caski and CSCC1 when compared with normal KCs. RelA K310 acetylation was lower in all three cervical cancer cell lines than in uninfected KCs (Fig. 5a; Supplementary Fig. 2c). For the Caski and CSCC1 lines this may be explained by the presence of upregulated IFRD1. However, the lack of RelA K310 acetylation in the CSCC7 line indicates that, besides IFRD1 also other mechanisms can alter the acetylation of RelA K310 in these squamous cancer cells.

Because IFRD1 was upregulated in the Caski and CSCC1 cells, we studied the effects of IFRD1 using these cell lines. IFRD1 knockdown in the CSCC1 cells did not alter basal cytokine expression levels (Supplementary Fig. 5a), but IFRD1 knockdown in the Caski cells resulted in a direct increase of the basal expression levels of CCL2 and RANTES (Fig. 5b). Furthermore, both cell lines showed increased cytokine gene levels upon IFN- $\gamma$ and TNF- $\alpha$ stimulation when IFRD1 was knocked down as compared with their control knockdown counterparts (Fig. 5c; Supplementary Fig. 5b).

CSCC1 and Caski cells express EGFR (Fig. 5d) at a level that is similar to that of uninfected KCs (Fig. 5e). However, the downstream signalling pathway is known to be constitutively higher in HPV-induced cancer cells ${ }^{32}$. As a consequence, the treatment of Caski and CSCC1 cancer cells with the anti-EGFR antibody cetuximab resulted in a higher production of IFN- $\gamma$ and TNF- $\alpha$-induced cytokines than when the cancer cells were treated with the control anti-CD20 antibody rituximab (Fig. 5f; Supplementary Fig. 5c). The enhanced response to IFN- $\gamma$ and TNF- $\alpha$ was associated with a concomitant decrease in IFRD1 protein levels (Fig. 5g), but not mRNA expression (Fig. 5h), upon EGFR blockade. Similarly, treatment of Caski and CSCC1 cancer cells with entinostat resulted in a higher production of CCL2, IL-8 and CXCL9 by the cancer cells when stimulated with IFN- $\gamma$ and
TNF- $\alpha$ than dimethylsulphoxide carrier control-treated cells (Fig. 5i; Supplementary Fig. 5d). Congruent with our earlier observations, RANTES levels diminished after entinostat treatment. These results suggest that IFRD1 may also play a role in suppressing the response of cancer cells to immune stimuli such as IFN- $\gamma$ and TNF- $\alpha$.

\section{Discussion}

Using a unique in vitro model, we here show that hrHPV infection leads to the upregulated expression of endogenous IFRD1 to deregulate the K310 acetylation of NFKB/RelA. As a result, hrHPV-infected KCs display an impaired production of pro-inflammatory cytokines and chemokines, and a reduced capacity to attract immune cells. The increased expression of IFRD1 in hrHPV $+\mathrm{KCs}$ is mediated by EGFR signalling via mTOR, RAF and/or MEK1. Knockdown of IFRD1 with siRNA or indirectly via blockade of EGFR with the clinically used EGFRspecific antibody cetuximab, resulted in decreased IFRD1 mRNA and protein levels, increased NFKB/RelA K310 acetylation and enhanced expression and production of pro-inflammatory cytokines and chemokines by hrHPV + KCs. The use of entinostat indicated that HDAC1 and/or -3 are involved in lowering K310 acetylation of NFKB/RelA. These conclusions are schematically represented in Fig. 5j.

EGFR activation on epithelial cells has been shown to result in a decreased production of CCL2, RANTES and CXCL10 and increased production of IL-8. Inhibition of EGFR signalling with blocking antibodies or tyrosine kinase inhibitors can reverse the effect on these cytokines, as well as result in an increased epithelial immune infiltrate in vivo ${ }^{33-35}$. Interestingly, virusinduced EGFR activation has been implicated as a novel mechanism for respiratory viruses to suppress antiviral host responses ${ }^{33}$. The exact underlying mechanism on EGFRmediated immune suppression remained unclear, albeit that ERK1/2 signalling was shown to be involved in regulating cytokine production and skin inflammation ${ }^{36}$. Using the EGFRblocking antibody cetuximab in the absence of an additional EGFR stimulus such as the transforming growth factor- $\alpha$, we found similar effects on the cytokine production of HPV16 + KCs. In KCs, the expression of EGFR and IFRD1 are tightly linked, as EGFR inhibition reduced the expression and protein levels of IFRD1, via mTOR, RAF and/or MEK1, but not PI3K or JNK. This fits with the involvement of ERK $1 / 2$ in regulating cytokine production ${ }^{36}$, since RAF and MEK1 are just upstream of these kinases. On the basis of our data, the previously observed EGFR activation-induced suppression of cytokine production and immune cell infiltration of epithelia can be explained by upregulation of IFRD1 and subsequent suppression of NFKB signalling. Our data suggest that EGFR-driven overexpression of IFRD1 may also play a role in deregulating $\mathrm{NF \kappa B}$-signalling in $\mathrm{HPV}$-induced tumour cells. Knockdown of

Figure 3 | Blocking EGFR signalling decreases IFRD1 levels and rescues cytokine production by hrHPV + KCs. (a) Microarray intensities for EGFR in $\mathrm{KCs}(n=4)$ and hrHPV $+\mathrm{KCs}(n=4)$ represented in a box plot. (b) Histogram of EGFR surface protein expression on KCs and HPV16 + KCs, as determined by flow cytometry. (c) RT-qPCR of EGFR expression in KCs transfected with complementary DNA for E2, E5, E1 + E2 + E6 + E7 or empty control. (d) RT-qPCR of IFRD1 expression in KCs and HPV16 + KCs treated for $72 \mathrm{~h}$ with $0,0.1,1$ or $10 \mu g \mathrm{ml}^{-1}$ anti-EGFR or anti-CD20. (e) IFRD1, RelA $\mathrm{K} 310$ acetylation and total RelA levels in KCs and HPV16 $+\mathrm{KCs}$ treated for $72 \mathrm{~h}$ with $0,0.1,1$ or $10 \mu \mathrm{g} \mathrm{ml} \mathrm{I}^{-1}$ anti-EGFR or anti-CD20. (f) Quantified protein levels of IFRD1, RelA K310 acetylation and RelA over $\beta$-actin in HPV16 + KCs treated for $72 \mathrm{~h}$ with 0, $0.1,1$ or $10 \mu \mathrm{g} \mathrm{ml} \mathrm{I}^{-1}$ anti-EGFR (two-dimensional western blot). The expression levels of the $0 \mu \mathrm{g} \mathrm{ml} \mathrm{m}^{-1}$-treated HPV $+\mathrm{KCs}$ were set as $100 \%$. (g) RT-qPCR of CCL2, RANTES, IL-8 and CXCL9 expression in 24-h non- or IFN- $\gamma$ - and TNF- $\alpha$-stimulated, anti-CD20- or anti-EGFR-treated HPV16 + KCs (left) and KCs (right). (h) Enzyme-linked immunosorbent assay for CCL2, RANTES, IL-8 and CXCL9 in cleared supernatants of 24-h non- or IFN- $\gamma$ - and TNF- $\alpha$-stimulated, anti-CD20- or anti-EGFR-treated HPV16 + KCs (left) and KCs (right). (i) RT-qPCR of IFRD1 expression in HPV16 + KCs treated with inhibitors of PI3K (LY94002, $25 \mu \mathrm{M})$, mTOR (rapamycin, $50 \mathrm{nM}$ ), MEK1 (PD98059, $50 \mu \mathrm{M}$ ), RAF (GW5074, $20 \mu \mathrm{M}$ ) and JNK (SP60025, $20 \mu \mathrm{M}$ ). Gene expression was normalized using GAPDH as the calibrator gene. Fold changes over control were calculated and depicted. These data are representative for at least three independent experiments, except for $\mathbf{h}$ that was performed once. Error bars indicate s.d. $P$ values were determined using Welch-corrected unpaired $t$-tests. ${ }^{\star} P<0.05,{ }^{\star \star} P<0.01$ and ${ }^{\star \star \star} P<0.001$. 
IFRD1 results in an increased production of pro-inflammatory cytokines and chemokines by tumour cells when stimulated with IFN- $\gamma$ and TNF- $\alpha$. Furthermore, blocking of the EGFR by cetuximab resulted in a decrease of IFRD1 protein levels, as well as increased cytokine production. The HPV oncoproteins are also known to directly intervene with NFKB signalling. Studies with transfected or transformed cells-resembling protein expression in tumour cells-show that E6 and/or E7 proteins inhibit basal
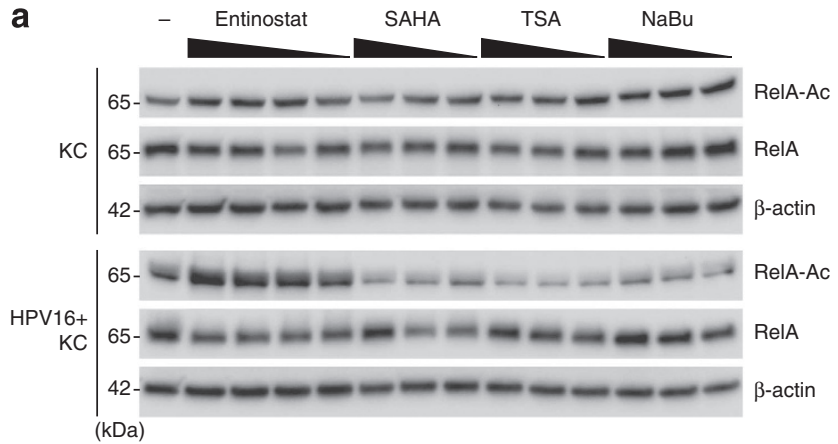

b Basal expression in

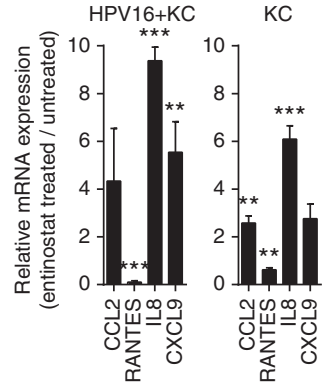$$
\text { (1) }
$$

C
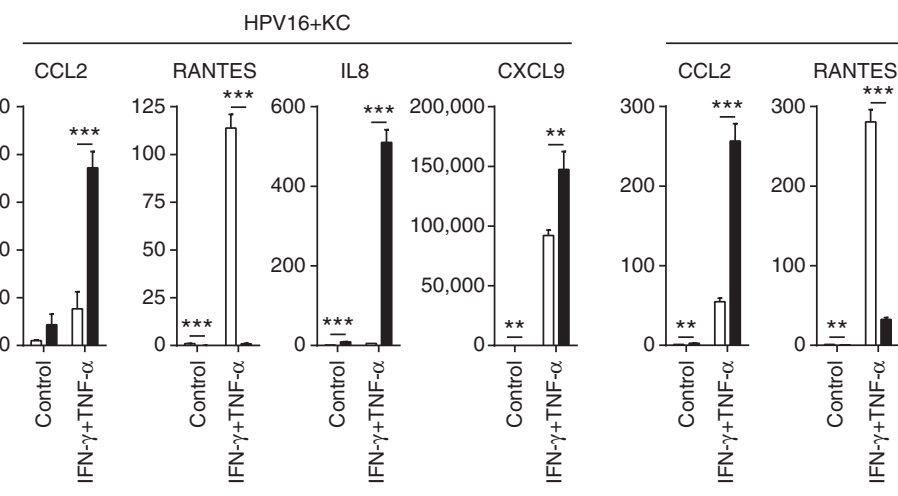

$\mathrm{KC}$

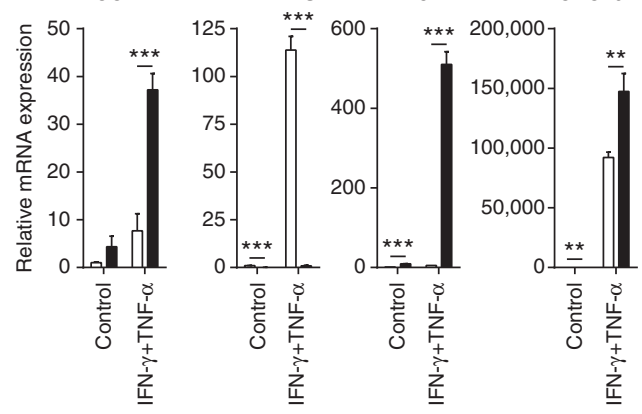

d

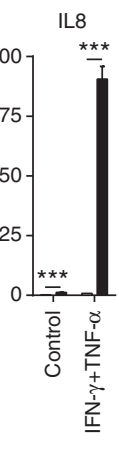

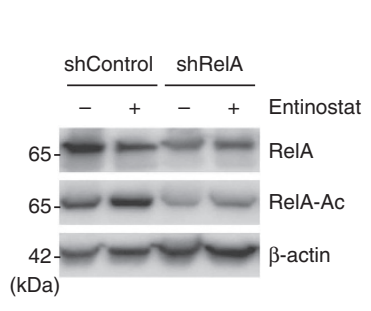
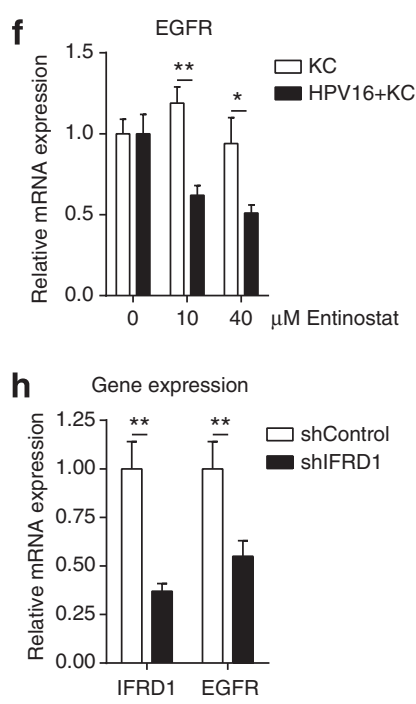

e

$$
\text { CCL2 }
$$

RANTES
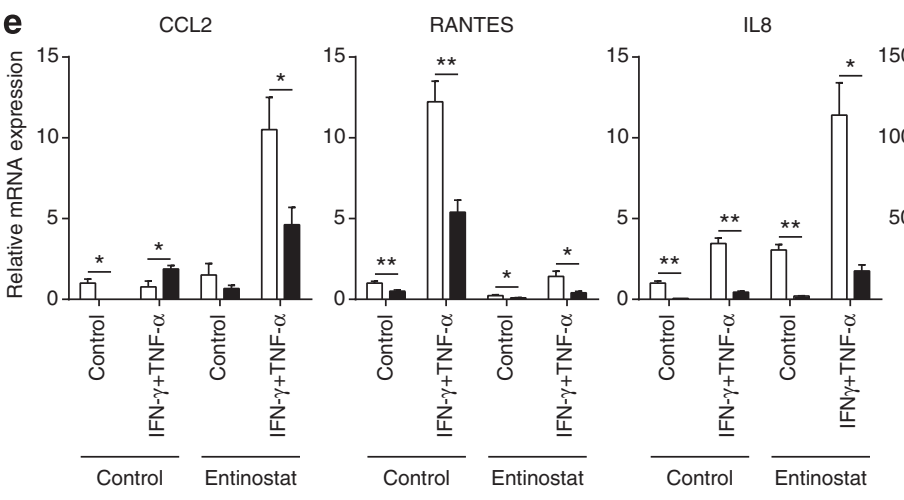

g
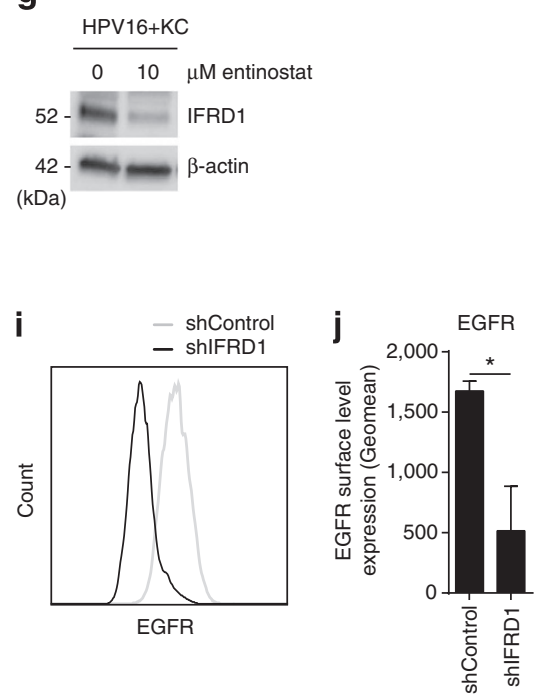


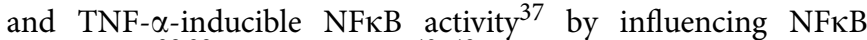
localization $^{38,39}$ and activation ${ }^{40-43}$.

Studies in immunosuppressed patients and healthy individuals show a key role for the adaptive immune response, in particular that of a strong type-1 (IFN- $\gamma$ and TNF- $\alpha$ )-associated HPV early antigen-specific $\mathrm{T}$ cells in the protection against progressive disease ${ }^{5}$. This notion is sustained by the clinical responses of patients treated with HPV-specific therapeutic vaccines ${ }^{5}$. Ample reasons, therefore, for HPV to also develop strategies preventing KCs to respond to these cytokines. Our data show that HPV deploys multiple strategies to interfere with induced RelA-associated NFKB signalling. HPV utilizes the cellular deubiquitinase UCHL1 to interfere with TRAF3, TRAF6 and NEMO function ${ }^{8}$, and here we show that HPV also upregulates the expression of endogenous IFRD1 to deregulate the K310 acetylation of NFKB. Furthermore, the E7 protein of hrHPV has been shown to bind HDAC1 and prevent acetylation of histones, thereby suppressing TLR9 signaling ${ }^{44}$, but E7 can also displace HDACs resulting in enhanced hypoxia-inducible factor- $1 \alpha$ transcriptional activity ${ }^{45}$. It is not unusual for viruses to target NFKB activation ${ }^{46,47}$, and hampering RelA acetylation is a common strategy. For instance, the $\mathrm{N}$ terminus of the orf virus protein 002 inhibits acetylation of RelA by blocking phosphorylation of RelA S276 and subsequent recruitment of acetylases $\mathrm{p} 300$ and $\mathrm{CBP}^{48}$, and the A238L protein of the African swine fever virus hampers RelA K310 acetylation by inhibiting RelA-p300 interaction ${ }^{49}$. We here postulate that hrHPV does not hamper KATs in acetylating RelA, but rather recruits a mediator to enhance HDAC-mediated RelA deacetylation. Together with our observation that HPV lowers basal cytokine expression in resting KCs due to the presence of IFRD1, we suggest that impairment of immune-driven RelA-associated NFkB-responsive gene expression is crucial for the virus to persist. This viral strategy has not been reported before, but as discussed above may also be employed by respiratory viruses that activate EGFR ${ }^{33}$.

All together, our data indicate that HPV upregulates EGFR to drive IFRD1 expression as a tool to decrease basal and adaptiveimmune system-driven cytokine expression. This may allow hrHPV to evade the host's immune response. It is highly likely that this mechanism plays a role in other viral infections too and even extends to tumours.

\begin{abstract}
Methods
Ethics statement. The use of discarded human foreskin, cervical and vaginal KC tissues to develop cell lines for these studies was approved by the institutional review board at the Pennsylvania State University College of Medicine and by the institutional review board at Pinnacle Health Hospitals. The Medical Ethical Committee of the Leiden University Medical Center approved the human tissue sections (healthy foreskin, healthy cervix and HPV16- or 18-positive cervical neoplasias) used for staining. All sections and cell lines were derived from discarded tissues and de-identified, therefore no informed consent was necessary.
\end{abstract}

Cell culture. Primary cultures of human epithelial KCs were established from foreskin, vaginal, vulva and cervical tissues, as previously described ${ }^{3}$, and grown in
KC serum-free medium (Medium 154 supplemented with the HKGS kit, Invitrogen, Breda, The Netherlands). KCs stably maintaining the full episomal $\mathrm{HPV}$ genome following electroporation (HPV-positive $\mathrm{KCs}$ ) were grown in monolayer culture using E-medium in the presence of mitomycin C (SigmaAldrich, Zwijndrecht, The Netherlands)-treated J2 3T3 feeder cells ${ }^{19,20}$ for two passages and were then adapted to KC serum-free medium for one passage before experimentation. J2 3T3 mouse fibroblasts, Caski, CSCC1, CSCC7 and SiHa cell lines were cultured in Iscove's modified Dulbecco's medium (IMDM) supplemented with $8 \%$ fetal bovine serum, $2 \mathrm{mM} \mathrm{l-glutamine}$ and $1 \%$ penicillinstreptomycin (complete IMDM medium) (Gibco-BRL, Invitrogen).

HPV16 infection of non-infected KCs. Primary basal layer human foreskin KCs were seeded at 75,000 cells per well in 24-well plates and allowed to attach for $48 \mathrm{~h}$. Cells received fresh medium (mock infected) or medium containing native HPV16 isolated from raft cultures at multiplicity of infection 100 for $24 \mathrm{~h}$. Cells were washed and harvested for either RT-qPCR or western blotting analysis.

IFRD1 and RelA knockdown in HPV-positive KCs. shRNAs were obtained from the MISSION TRC-library of Sigma-Aldrich. The MISSION shRNA clones are sequence-verified shRNA lentiviral plasmids (pLKO.1-puro) provided as frozen bacterial glycerol stocks (Luria broth, carbenicillin at $100 \mu \mathrm{g} \mathrm{ml} l^{-1}$ and $10 \%$ glycerol) in Escherichia coli for propagation and downstream purification of the shRNA clones. pLKO.1 contains the puromycin selection marker for transient or stable transfection. The construct against IFRD1 (NM_001550) was

TRCN0000156194: 5' -CCGGCAGTTCTGAAACAGTTTCTTTCTCGAGAAAGA AACTGTTTCAGAACTGTTTTT-3', RelA (NM_021975) was TRCN0000014687: 5'-CCGGCCTGAGGCTATAACTCGCCTACTCGAGTAGGCGAGTTATAGCCT CAGGTTTTT- $3^{\prime}$ and the control was: SHC004 (MISSION TRC2-pLKO puro TurboGFP shRNA control vector): $5^{\prime}$-CCGGCGTGATCTTCACCGACAAGAT CTCGAGATCTTGTCGGTGAAGATCACGTTTTT-3'. HPV16-positive KCs at $\sim 60 \%$ confluence were transduced with lentivirus at multiplicity of infection 5-10 overnight, after which the medium was replaced. At least $72 \mathrm{~h}$ post transduction, cells were stimulated as indicated and target gene expression was assayed by RT-qPCR or western blotting.

HPV knockdown in HPV-positive KCs. Silencer Select siRNA against HPV16 E2 (5'-AACACUACACCCAUAGUACAUtt- $\left.3^{\prime}\right)$ was designed using siRNA Target Finder software (Ambion, Invitrogen). Blast search revealed that the designed E2 siRNA does not match with the known human transcriptome. E2 and negative control \#2 siRNA (sequence not provided by the manufacturer) were purchased from Ambion. HPV16 + KCs were transfected with $50 \mathrm{nM}$ siRNA E2 or negative control \#2 using Lipofectamine 2000 (Invitrogen), according to the manufacturer's instructions. Forty-eight hours post transfection, cells were harvested or stimulated as indicated and target gene expression was assayed by RT-qPCR or western blotting.

Transfection of HPV genes into non-infected KCs. Non-infected primary KCs were seeded at 50,000 cells per well in 24-well plates and allowed to attach overnight. Cells were transfected with $500 \mathrm{ng}$ DNA using Lipofectamine (Invitrogen), according to the manufacturer's instructions. Cells were maintained in E-medium. Seventy-two hours post transfection, cells were harvested and target gene expression was assayed by RT-qPCR.

EGFR signalling blocking. Subconfluent cells were cultured in respective complete growth medium in the presence of cetuximab $\left(0.1,1\right.$ or $10 \mu \mathrm{g} \mathrm{ml}^{-1}$; Merck serono) rituximab (0.1, 1 or $10 \mu \mathrm{g} \mathrm{ml}^{-1}$; Roche), rapamycin ( $50 \mathrm{nM}$; Calbiochem),

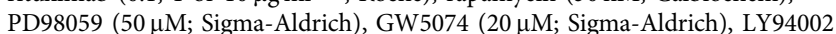
$(25 \mu \mathrm{M}$; Sigma-Aldrich) or SP60025 (20 $\mu \mathrm{M}$; Sigma-Aldrich). Medium was changed every $2-3$ days. After at least $72 \mathrm{~h}$, cells were stimulated as indicated and target gene expression was assayed by RT-qPCR or western blotting.

Figure 4 | Entinostat treatment reveals involvement of HDAC1/3 in RelA deacetylation in HPV16 + KCs. (a) RelA K310 acetylation and total RelA levels in KCs and HPV16 + KCs treated with decreasing doses of entinostat (40,20, 10 and $2 \mu \mathrm{M}), \mathrm{SAHA}(10,5$ and $1 \mu \mathrm{M}), \mathrm{TSA}(5,1$ and $0.333 \mu \mathrm{M})$ or NaBu $(10,5$ and $1 \mathrm{mM})$. RT-qPCR of CCL2, RANTES, IL-8 and CXCL9 expression in steady state (b) or 24-h non- or IFN- $\gamma$ - and TNF- $\alpha$-stimulated (c) control or entinostat $(10 \mu \mathrm{M})$ pre-treated HPV16 + KCs. (d) Total RelA levels and RelA K310 acetylation in non- or entinostat-treated control or RelA knockdown (KD) HPV16 + KCs. (e) RT-qPCR of CCL2, RANTES, IL-8 and CXCL9 expression in 24-h non- or IFN- $\gamma$ - and TNF- $\alpha$-stimulated non- or entinostat-treated control or RelA knockdown (KD) HPV16 + KCs. (f) RT-qPCR of EGFR expression in KCs and HPV16 + KCs treated with increasing doses of entinostat (0, 10 or $40 \mu \mathrm{M}$ ). Gene expression was normalized using GAPDH as the calibrator gene. (g) IFRD1 in control or entinostat $(10 \mu \mathrm{M})$ pre-treated HPV16 + KCs. (h) RT-qPCR of IFRD1 and EGFR expression in control or IFRD1 KD HPV16 + KCs. Gene expression was normalized using GAPDH as the calibrator gene. Histogram (i) and geomean (j) of EGFR expression on control or IFRD1 KD HPV16 + KCs, as determined by flow cytometry. s.e.m. of two independent experiments. These data are representative for at least two independent experiments. Error bars indicate s.d. $P$ values were determined using Welch-corrected unpaired $t$-tests. ${ }^{\star} P<0.05,{ }^{\star \star} P<0.01$ and ${ }^{\star \star \star} * P<0.001$. 
a

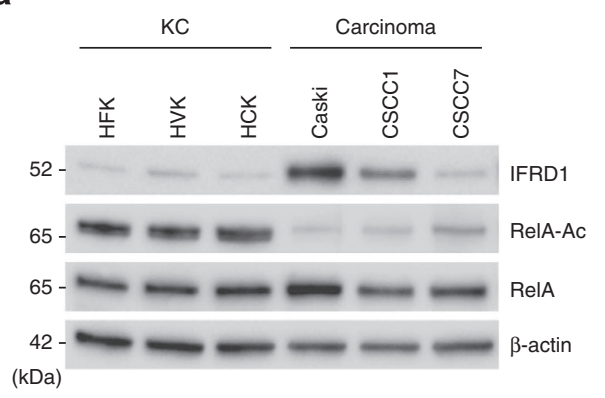

b

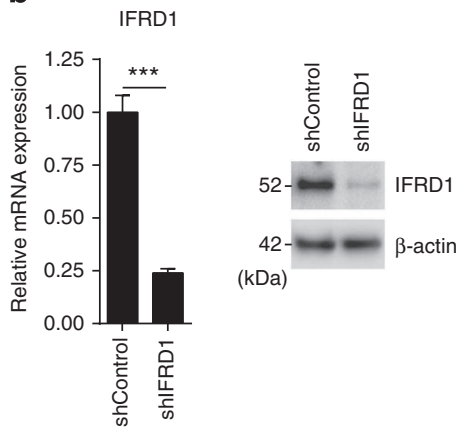

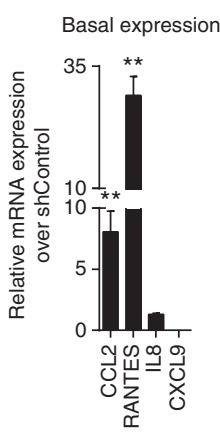

e

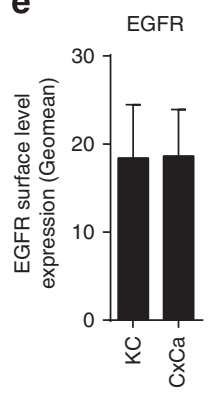

Unstained

- $\csc 1$
C

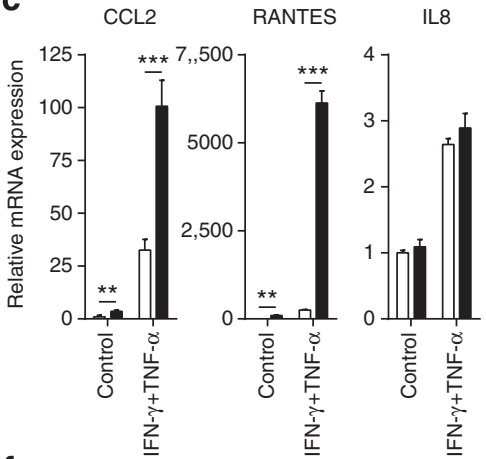

f

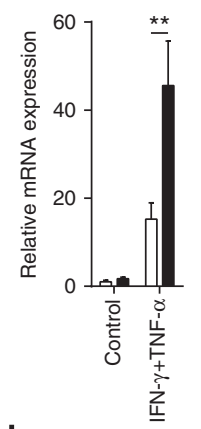

RANTES

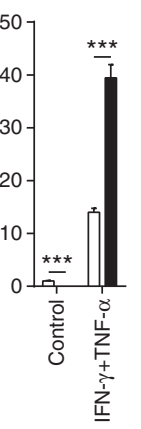

แL8

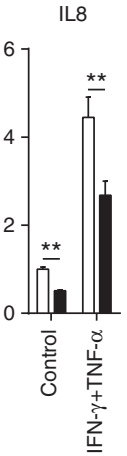

CXCL9

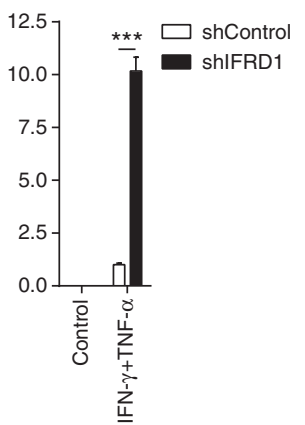

CXCLI

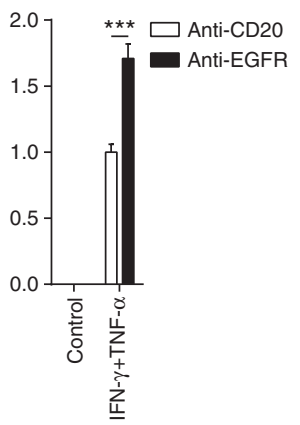

d

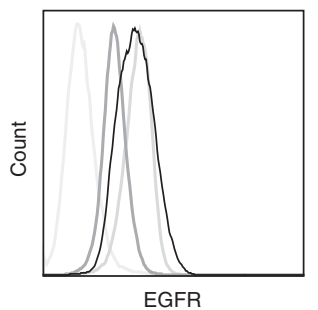

- $\operatorname{CscC7}$

- Caski

g

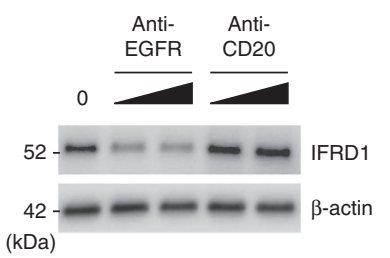

h IFRD1

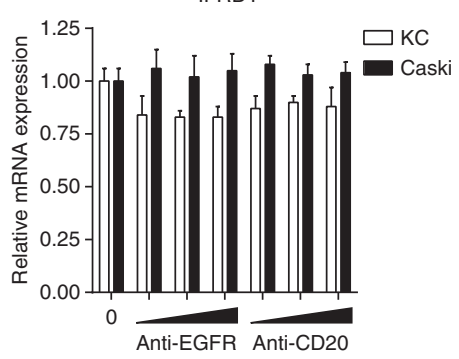

i
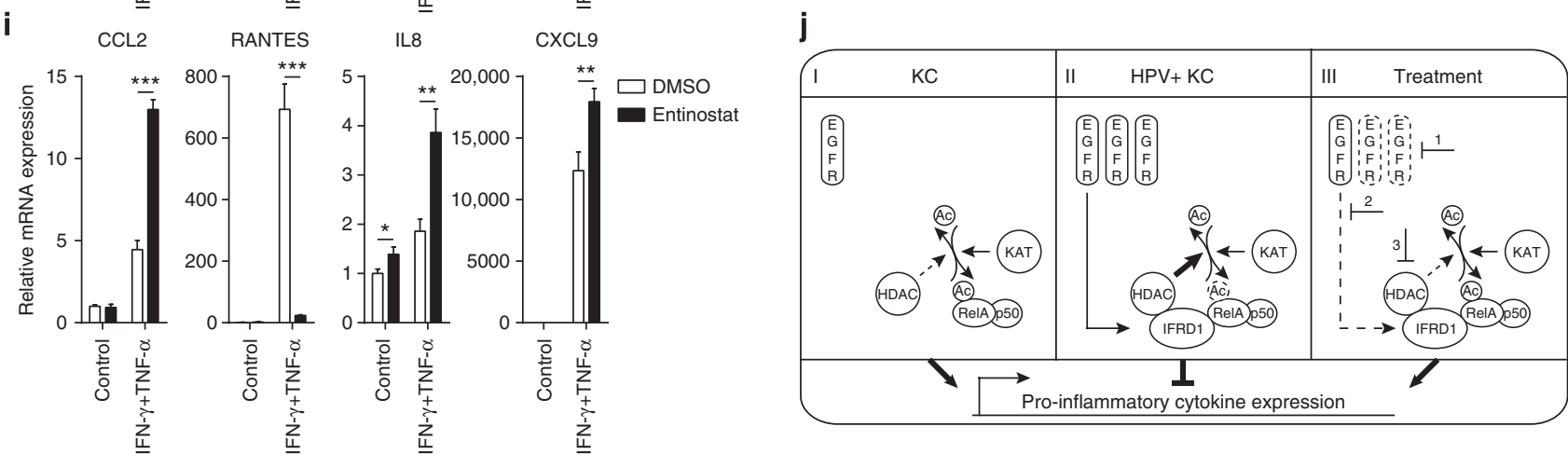

Figure $\mathbf{5}$ | Role of IFRD1 in hrHPV + cervical cancer cells. (a) IFRD1, RelA acetylation and total RelA levels at steady state in three KC donors and three HPV16-induced CXCa lines. (b) RT-qPCR of IFRD1, CCL2, RANTES, IL-8 and CXCL9 expression, and IFRD1 protein levels in steady-state control or IFRD1 KD Caski cells. (c) RT-qPCR of CCL2, RANTES, IL-8 and CXCL9 expression in 24-h non- or IFN- $\gamma$ - and TNF- $\alpha$-stimulated control or IFRD1 KD Caski cells. (d) Histogram of EGFR expression on three HPV16-induced CxCa lines. (e) Geomean of EGFR expression on KCs and CxCa, as determined by flow cytometry. s.e.m. of two independent experiments. (f) RT-qPCR of CCL2, RANTES, LL-8 and CXCL9 expression in 24-h non- or IFN- $\gamma$ - and TNF- $\alpha$-stimulated anti-CD2O- or anti-EGFR-treated Caski cells. (g) IFRD1 and RelA K310 acetylation status in Caski cells treated for $72 \mathrm{~h}$ with 0,1 or $10 \mu \mathrm{g} \mathrm{ml} \mathrm{I}^{-1}$ anti-EGFR (cetuximab) or anti-CD20 (rituximab). (h) RT-qPCR of IFRD1 expression in KCs and Caski cells treated for $72 \mathrm{~h}$ with 0, 0.1, 1 or $10 \mu \mathrm{g} \mathrm{ml} \mathrm{I}^{-1}$ anti-EGFR or anti-CD20. (i) RT-qPCR of CCL2, RANTES, IL-8 and CXCL9 expression in 24-h non- or IFN- $\gamma$ - and TNF- $\alpha$-stimulated control (dimethylsulphoxide (DMSO)) or entinostat-treated Caski cells. (j) Schematic representation of IFRD1-mediated RelA (de-)acetylation. (I) In KCs, RelA acetylation is positively regulated by KATs, resulting in the production of pro-inflammatory cytokines. HDACs may suppress this process. (II) In HPV + KCs, elevated EGFR levels can induce the expression of IFRD1, which can mediate RelA deacetylation by forming a bridge between RelA and HDAC1 and/or -3, hampering pro-inflammatory gene expression. (III) Interfering with EGFR signalling (1 and 2) or HDAC function (3) may lower IFRD1 levels, restoring the RelA acetylation balance, augmenting pro-inflammatory gene expression. Error bars indicate s.d. $P$ values were determined using Welch-corrected unpaired $t$-tests. ${ }^{\star} P<0.05,{ }^{\star \star} P<0.01$ and ${ }^{\star \star \star} P<0.001$. 
HDAC inhibition. Subconfluent cells were cultured in presence of a dilution series of entinostat (MS-175; 40,20, 10 and $2 \mu \mathrm{M}$; Selleckchem BioConnect), vorinostat (SAHA; 10, 5 and $1 \mu \mathrm{M}$; Sigma-Aldrich), trichostatin A (5, 1 and $0.333 \mu \mathrm{M}$; SigmaAldrich) or sodium butyrate (NaBu; 10, 5 and $1 \mathrm{mM}$; Sigma-Aldrich) in the respective complete growth medium overnight. Medium was changed for the respective complete growth medium, cells were stimulated as indicated and target gene expression was assayed by RT-qPCR or western blotting. Since treatment with $10 \mu \mathrm{M}$ entinostat showed a good increase in RelA K310 acetylation without signs of toxicity, subsequent experiments were performed using this dose.

Migration assays. (HPV-positive) KCs were stimulated as indicated for $24 \mathrm{~h}$. Cleared (HPV-positive) KC supernatants were added to the lower compartment of a transwell plate (Corning). The upper compartment was filled with PBMCs isolated from buffy coats (Sanquin). PBMCs were allowed to migrate for $16 \mathrm{~h}$, after which the cells in the lower compartment were counted by flow cytometry in the presence of counting beads (Invitrogen), according to the manufacturer's instructions. Myeloid cells and lymphocytes were differentiated by their respective size in the Forward Scatter (FSC)/Side Scatter (SSC) plot (data not shown).

RNA expression analyses and ELISA. The microarray data ${ }^{12}$ are accessible in the Gene Expression Omnibus database (accession number GSE54181). Plots were generated using the webtool R2: microarray analysis and visualization platform (http://r2.amc.nl).

Total RNA was isolated using the NucleoSpin RNA II kit (Machery-Nagel, Leiden, The Netherlands) according to the manufacturer's instructions. Total RNA $(0.5-1.0 \mu \mathrm{g})$ was reverse transcribed using the SuperScript III First Strand synthesis system from Invitrogen. TaqMan PCR was performed using the TaqMan Universal PCR Master Mix and pre-designed, pre-optimized primers and probe mix for CCL2, RANTES (CCL5), IL-8 (CXCL8), CXCL9 and GAPDH (Applied Biosystems, Foster City, USA). Threshold cycle numbers (Ct) were determined using the CFX PCR System (Bio-Rad, Veenendaal, The Netherlands), and the relative quantities of complementary DNA per sample were calculated using the $\Delta \Delta \mathrm{Ct}$ method using GAPDH as the calibrator gene.

Enzyme-linked immunosorbent assays (ELISAs) for CCL2, RANTES, IL-8 and CXCL9 were performed according to the manufacturer's instruction (PeproTech, London, UK)

Flow cytometry. Expression of EGFR on KCs was analysed by flow cytometry using phycoerythrin (PE)-coupled mouse-anti-human EGFR (1:20, BD Biosciences, Breda, The Netherlands). Per live gate, 50,000 cells were recorded using the BD FACS Calibur with Cellquest software (BD Bioscience) and data were analysed using Flowjo (Treestar, Olten, Switzerland).

Western blot analysis. For western blotting, polypeptides were resolved by SDS-polyacrylamide gel electrophoresis and transferred to a nitrocellulose membrane (Bio-Rad). Immunodetection was achieved with anti-p65 (1:1,000, sc-372, Santa Cruz), anti-phospho-p65 (Ser536; 1:1,000, \#3033 Cell Signaling Technology (CST)), anti-acetyl-p65 (Lys310; 1:1,000, \#3045 CST), anti-IFRD1 (1:400, T2576 Sigma-Aldrich), $\beta$-actin (1:10,000, Sigma-Aldrich) primary antibodies, and horseradish peroxidase-coupled anti-mouse (1:5,000; CST) and horseradish peroxidase-coupled anti-rabbit $(1: 5,000$, CST) secondary antibodies. Chemoluminescence reagent (Bio-Rad) was used as a substrate and the signal was scanned using the Chemidoc and accompanying software (Bio-Rad) to quantify the intensity of the bands as a measure of the amount of protein of interest in the blot. The relative amount was determined by calculating the ratio of each protein over that of the density measured for the housekeeping protein $\beta$-actin. Images have been cropped for presentation. Full-size images are presented in Supplementary Fig. 6.

Immunohistochemistry. Four $\mu \mathrm{m}$ formalin-fixed, paraffin-embedded tissue sections from two random vulvar intraepithelial neoplasia cases were deparaffinized and rehydrated using graded concentrations of ethanol to distilled water. Endogenous peroxidise activity was blocked with $0.03 \% \mathrm{H}_{2} \mathrm{O}_{2} / \mathrm{MeOH}$ for $20 \mathrm{~min}$. Antigen retrieval was performed in boiling EDTA buffer ( $\mathrm{pH} 9.0)$ for $12 \mathrm{~min}$. After $2 \mathrm{~h}$ of cooling down to room temperature, slides were washed twice in distilled water and twice in PBS. Subsequently, incubation was performed overnight at room temperature with the primary IFRD1 antibody (T2576 Sigma-Aldrich; 1:500 in PBS containing $1 \%$ bovine serum albumin); p16 (CINTEC, diluted 1:5) and E2 (1:50) (provided by Dr F. Thierry). Second, sections were incubated with BrightVision polyhorseradish peroxidase anti-mouse/rabbit/rat immunoglobulin-G (Immunologic BV, Duiven, The Netherlands) for $30 \mathrm{~min}$ at room temperature. Washing between incubations was performed three times for $5 \mathrm{~min}$ in PBS. Immune complexes were visualized by applying a $0.05-\mathrm{M}$ tris- $\mathrm{HCl}$ buffer ( $\mathrm{pH} 7.6)$ containing $0.05 \%$ of $3,3^{\prime}$-diamino-benzidine-tetrahydrochloride and $0.0018 \%$ of $\mathrm{H}_{2} \mathrm{O}_{2}$. After $10 \mathrm{~min}$, the reaction was stopped by rinsing with demineralized water. Finally, the tissue sections were counterstained with Mayer's haematoxylin before addition of a coverslip.
Statistical analysis. Statistical analysis was performed using GraphPad InStat version 3.00. $P$ values were determined using Welch-corrected unpaired $t$-tests. ${ }^{*} P<0.05,{ }^{* *} P<0.01$ and ${ }^{* *} P<0.001$.

\section{References}

1. Doorbar, J. Molecular biology of human papillomavirus infection and cervical cancer. Clin. Sci. 110, 525-541 (2006).

2. Frazer, I. H. Interaction of human papillomaviruses with the host immune system: a well evolved relationship. Virology 384, 410-414 (2009).

3. Karim, R. et al. Human papillomavirus deregulates the response of a cellular network comprising of chemotactic and proinflammatory genes. PLOS ONE 6, e17848 (2011).

4. Richardson, H. et al. The natural history of type-specific human papillomavirus infections in female university students. Cancer Epidemiol. Biomarkers Prev. 12, 485-490 (2003).

5. van der Burg, S. H. \& Melief, C. J. Therapeutic vaccination against human papilloma virus induced malignancies. Curr. Opin. Immunol. 23, 252-257 (2011)

6. zur Hausen, H. Papillomaviruses and cancer: from basic studies to clinical application. Nat. Rev. Cancer 2, 342-350 (2002).

7. Hasan, U. A. et al. TLR9 expression and function is abolished by the cervical cancer-associated human papillomavirus type 16. J. Immunol. 178, 3186-3197 (2007).

8. Karim, R. et al. Human papillomavirus (HPV) upregulates the cellular deubiquitinase UCHL1 to suppress the keratinocyte's innate immune response. PLoS Pathog. 9, e1003384 (2013).

9. Reiser, J. et al. High-risk human papillomaviruses repress constitutive kappa interferon transcription via E6 to prevent pathogen recognition receptor and antiviral-gene expression. J. Virol. 85, 11372-11380 (2011).

10. Sunthamala, N. et al. E2 proteins of high risk human papillomaviruses down-modulate STING and IFN-kappa transcription in keratinocytes. PLoS ONE 9, e91473 (2014).

11. Termini, L. et al. Characterization of global transcription profile of normal and HPV-immortalized keratinocytes and their response to TNF treatment. BMC Med. Genomics 1, 29 (2008).

12. Tummers, B. et al. CD40-mediated amplification of local immunity by epithelial cells is impaired by HPV. J. Invest. Dermatol. 134, 2918-2927 (2014)

13. Chang, Y. E. \& Laimins, L. A. Microarray analysis identifies interferoninducible genes and Stat-1 as major transcriptional targets of human papillomavirus type 31. J. Virol. 74, 4174-4182 (2000).

14. Hong, S., Mehta, K. P. \& Laimins, L. A. Suppression of STAT-1 expression by human papillomaviruses is necessary for differentiation-dependent genome amplification and plasmid maintenance. J. Virol. 85, 9486-9494 (2011).

15. Nees, M. et al. Papillomavirus type 16 oncogenes downregulate expression of interferon-responsive genes and upregulate proliferation-associated and NF-kappaB-responsive genes in cervical keratinocytes. J. Virol. 75, 4283-4296 (2001).

16. Zhou, F., Chen, J. \& Zhao, K. N. Human papillomavirus 16 -encoded E7 protein inhibits IFN-gamma-mediated MHC class I antigen presentation and CTL-induced lysis by blocking IRF-1 expression in mouse keratinocytes. J. Gen Virol. 94, 2504-2514 (2013).

17. Chen, L. F. \& Greene, W. C. Shaping the nuclear action of NF-kappaB. Nat. Rev. Mol. Cell Biol. 5, 392-401 (2004).

18. Conway, M. J. \& Meyers, C. Replication and assembly of human papillomaviruses. J. Dent. Res. 88, 307-317 (2009).

19. McLaughlin-Drubin, M. E., Christensen, N. D. \& Meyers, C. Propagation, infection, and neutralization of authentic HPV16 virus. Virology 322, 213-219 (2004).

20. Meyers, C., Mayer, T. J. \& Ozbun, M. A. Synthesis of infectious human papillomavirus type 18 in differentiating epithelium transfected with viral DNA. J. Virol. 71, 7381-7386 (1997).

21. Lee, D., Lee, B., Kim, J., Kim, D. W. \& Choe, J. cAMP response element-binding protein-binding protein binds to human papillomavirus E2 protein and activates E2-dependent transcription. J. Biol. Chem. 275, 7045-7051 (2000).

22. Quinlan, E. J., Culleton, S. P., Wu, S. Y., Chiang, C. M. \& Androphy, E. J. Acetylation of conserved lysines in bovine papillomavirus E2 by p300. J. Virol. 87, 1497-1507 (2013).

23. Gu, Y. et al. Identification of IFRD1 as a modifier gene for cystic fibrosis lung disease. Nature 458, 1039-1042 (2009).

24. Micheli, L. et al. PC4/Tis7/IFRD1 stimulates skeletal muscle regeneration and is involved in myoblast differentiation as a regulator of MyoD and NF-kappaB. J. Biol. Chem. 286, 5691-5707 (2011).

25. Xue, Y. et al. HPV16 E2 is an immediate early marker of viral infection, preceding E7 expression in precursor structures of cervical carcinoma. Cancer Res. 70, 5316-5325 (2010).

26. von Knebel Doeberitz, M., Gissmann, L. \& zur Hausen, H. Growth-regulating functions of human papillomavirus early gene products in cervical cancer cells acting dominant over enhanced epidermal growth factor receptor expression. Cancer Res. 50, 3730-3736 (1990). 
27. Vietor, I. \& Huber, L. A. Role of TIS7 family of transcriptional regulators in differentiation and regeneration. Differentiation 75, 891-897 (2007).

28. Kim, M. K. et al. Human papillomavirus type 16 E5 oncoprotein as a new target for cervical cancer treatment. Biochem. Pharmacol. 80, 1930-1935 (2010).

29. Bruzzese, F. et al. HDAC inhibitor vorinostat enhances the antitumor effect of gefitinib in squamous cell carcinoma of head and neck by modulating ErbB receptor expression and reverting EMT. J. Cell. Physiol. 226, 2378-2390 (2011).

30. Liu, N. et al. Blocking the class I histone deacetylase ameliorates renal fibrosis and inhibits renal fibroblast activation via modulating TGF-beta and EGFR signaling. PLoS ONE 8, e54001 (2013).

31. Heusinkveld, M. et al. M2 macrophages induced by prostaglandin E2 and IL-6 from cervical carcinoma are switched to activated M1 macrophages by CD4 + Th1 cells. J. Immunol. 187, 1157-1165 (2011).

32. Feng, W., Duan, X., Liu, J., Xiao, J. \& Brown, R. E. Morphoproteomic evidence of constitutively activated and overexpressed mTOR pathway in cervical squamous carcinoma and high grade squamous intraepithelial lesions. Int. J. Clin. Exp. Pathol. 2, 249-260 (2009).

33. Kalinowski, A. et al. EGFR activation suppresses respiratory virus-induced IRF1-dependent CXCL10 production. Am. J. Physiol. Lung Cell. Mol. Physiol. 307, L186-L196 (2014).

34. Mascia, F., Mariani, V., Girolomoni, G. \& Pastore, S. Blockade of the EGF receptor induces a deranged chemokine expression in keratinocytes leading to enhanced skin inflammation. Am. J. Pathol. 163, 303-312 (2003).

35. Paul, T. et al. Cytokine regulation by epidermal growth factor receptor inhibitors and epidermal growth factor receptor inhibitor associated skin toxicity in cancer patients. Eur. J. Cancer 50, 1855-1863 (2014).

36. Pastore, S. et al. ERK1/2 regulates epidermal chemokine expression and skin inflammation. J. Immunol. 174, 5047-5056 (2005).

37. Vandermark, E. R. et al. Human papillomavirus type 16 E6 and E 7 proteins alter NF-kB in cultured cervical epithelial cells and inhibition of NF-kB promotes cell growth and immortalization. Virology 425, 53-60 (2012).

38. Caberg, J. H. et al. Increased migration of Langerhans cells in response to HPV16 E6 and E7 oncogene silencing: role of CCL20. Cancer Immunol. Immunother. 58, 39-47 (2009).

39. Havard, L., Rahmouni, S., Boniver, J. \& Delvenne, P. High levels of p105 (NFKB1) and p100 (NFKB2) proteins in HPV16-transformed keratinocytes: role of E6 and E7 oncoproteins. Virology 331, 357-366 (2005).

40. Avvakumov, N., Torchia, J. \& Mymryk, J. S. Interaction of the HPV E7 proteins with the PCAF acetyltransferase. Oncogene 22, 3833-3841 (2003).

41. Bernat, A., Avvakumov, N., Mymryk, J. S. \& Banks, L. Interaction between the HPV E7 oncoprotein and the transcriptional coactivator $\mathrm{p} 300$. Oncogene 22, 7871-7881 (2003)

42. Huang, S. M. \& McCance, D. J. Down regulation of the interleukin-8 promoter by human papillomavirus type $16 \mathrm{E} 6$ and E7 through effects on CREB binding protein/p300 and P/CAF. J. Virol. 76, 8710-8721 (2002).

43. Spitkovsky, D., Hehner, S. P., Hofmann, T. G., Moller, A. \& Schmitz, M. L. The human papillomavirus oncoprotein E7 attenuates NF-kappa B activation by targeting the Ikappa B kinase complex. J. Biol. Chem. 277, 25576-25582 (2002).

44. Hasan, U. A. et al. The human papillomavirus type $16 \mathrm{E} 7$ oncoprotein induces a transcriptional repressor complex on the Toll-like receptor 9 promoter. J. Exp. Med. 210, 1369-1387 (2013).
45. Bodily, J. M., Mehta, K. P. \& Laimins, L. A. Human papillomavirus E7 enhances hypoxia-inducible factor 1-mediated transcription by inhibiting binding of histone deacetylases. Cancer Res. 71, 1187-1195 (2011).

46. Hiscott, J., Nguyen, T. L., Arguello, M., Nakhaei, P. \& Paz, S. Manipulation of the nuclear factor-kappaB pathway and the innate immune response by viruses. Oncogene 25, 6844-6867 (2006).

47. Le Negrate, G. Viral interference with innate immunity by preventing NF-kappaB activity. Cell. Microbiol. 14, 168-181 (2012).

48. Ning, Z. et al. The $\mathrm{N}$ terminus of orf virus-encoded protein 002 inhibits acetylation of NF-kappaB p65 by preventing Ser(276) phosphorylation. PloS ONE 8, e58854 (2013).

49. Granja, A. G., Sabina, P., Salas, M. L., Fresno, M. \& Revilla, Y. Regulation of inducible nitric oxide synthase expression by viral A238L-mediated inhibition of p65/RelA acetylation and p300 transactivation. J. Virol. 80, 10487-10496 (2006).

\section{Acknowledgements}

This work was supported by the Netherlands Organization for Health Research (NOW/ZonMw) TOP grant 91209012. The funders had no role in study design, data collection and analysis, decision to publish or preparation of the manuscript.

\section{Author contributions}

B.T., R.G., E.S.J., C.J.M.M., J.M.B. and S.H.v.d.B. designed the experiments. B.T., R.G., L.P.L.P. and E.S.J. performed the experiments. L.P.L.P., E.M.G.M.v.E. and C.M. made viruses and cells. B.T. and S.H.v.d.B. wrote the paper. C.J.M.M., J.M.B. and S.H.v.d.B. supervised the project. All authors discussed the data.

\section{Additional information}

Supplementary Information accompanies this paper at http://www.nature.com/ naturecommunications

Competing financial interests: C.M. has received speaker honoraria from Merck, Quest Diagnostics, GSK and Bristol-Myers. C.M. has performed research funded by Merck, The Phillip Morris External Research Program, NexMed, GSK, OriGenix and Interferon Sciences Inc. The remaining authors declare no competing financial interests.

Reprints and permission information is available online at http://npg.nature.com/ reprintsandpermissions/

How to cite this article: Tummers, B. et al. The interferon-related developmental regulator 1 is used by human papillomavirus to suppress NFкB activation. Nat. Commun. 6:6537 doi: 10.1038/ncomms7537 (2015).

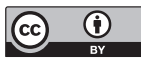

This work is licensed under a Creative Commons Attribution 4.0 International License. The images or other third party material in this article are included in the article's Creative Commons license, unless indicated otherwise in the credit line; if the material is not included under the Creative Commons license, users will need to obtain permission from the license holder to reproduce the material. To view a copy of this license, visit http://creativecommons.org/licenses/by/4.0/ 